MATHEMATICS OF COMPUTATION

Volume 76, Number 259, July 2007, Pages 1589-1617

S 0025-5718(07)01976-X

Article electronically published on February 19, 2007

\title{
QUADRATIC FORMS THAT REPRESENT ALMOST THE SAME PRIMES
}

\author{
JOHN VOIGHT
}

\begin{abstract}
Jagy and Kaplansky exhibited a table of 68 pairs of positive definite binary quadratic forms that represent the same odd primes and conjectured that their list is complete outside of "trivial" pairs. In this article, we confirm their conjecture, and in fact find all pairs of such forms that represent the same primes outside of a finite set.
\end{abstract}

\section{INTRODUCTION}

The forms $x^{2}+9 y^{2}$ and $x^{2}+12 y^{2}$ represent the same set of prime numbers, namely, those primes $p$ which can be written $p=12 n+1$ for some positive integer $n$. What other like pairs of forms exist? Jagy and Kaplansky JK performed a computer search for pairs that represent the same set of odd primes and found certain "trivial" pairs which occur infinitely often and listed other sporadic examples. They conjecture that their list is complete.

Using the tools of class field theory, in this article we give a provably complete list of such pairs. By a form $Q$ we mean an integral positive definite binary quadratic form $Q=a x^{2}+b x y+c y^{2} \in \mathbb{Z}[x, y]$; the discriminant of $Q$ is $b^{2}-4 a c=D=d f^{2}<0$, where $d$ is the discriminant of $\mathbb{Q}(\sqrt{D})$ or the fundamental discriminant, and $f \geq 1$. We will often abbreviate $Q=\langle a, b, c\rangle$.

Throughout, we look for forms that represent the same primes outside of a finite set-we then say that they represent almost the same primes. A form represents the same primes as any equivalent form under the action of the group $G L_{2}(\mathbb{Z})$. Hence from now on (except in the statement of Proposition 2.4, see Remark 2.5. and in the proof of Lemma (7.2), we insist that a form be $G L_{2}(\mathbb{Z})$-reduced, i.e., $0 \leq b \leq a \leq c$. Moreover, the set of primes represented by a form is finite (up to a finite set, it is empty) if and only if the form is nonprimitive, that is to say $\operatorname{gcd}(a, b, c)>1$, and any two nonprimitive forms represent almost the same primes. We therefore also insist that a form be primitive, so that the set of primes represented is infinite.

If $Q_{1}, Q_{2}$ are forms which represent almost the same primes, we write $Q_{1} \sim Q_{2}$; it is clear that $\sim$ defines an equivalence relation on the set of forms. To every equivalence class $C$ of forms, we associate the set $\delta(C)$ of fundamental discriminants $d$ of the forms in $C$ as well as the set $\Delta(C)$ of discriminants $D$ of forms in $C$.

Received by the editor September 16, 2005 and, in revised form, July 25, 2006.

2000 Mathematics Subject Classification. Primary 11E12; Secondary 11E16, 11R11.

Key words and phrases. Binary quadratic forms, number theory.

The author's research was partially supported by an NSF Graduate Fellowship. The author would like to thank Hendrik Lenstra, Peter Stevenhagen, and the reviewer for their helpful comments, as well as William Stein and the MECCAH cluster for computer time.

(C) 2007 American Mathematical Society Reverts to public domain 28 years from publication 
The main result of this article is the following (Theorem 6.2).

Theorem. There are exactly 67 equivalence classes $C$ of forms with $\# \delta(C) \geq 2$. There are exactly 6 classes with $\# \delta(C)=3$ and there is no class with $\# \delta(C) \geq 4$.

Corollary. There are exactly 111 pairs of forms $Q_{1}, Q_{2}$ with fundamental discriminants $d_{1} \neq d_{2}$ such that $Q_{1} \sim Q_{2}$.

The forms are listed in Tables $1-5$ at the end of this article.

As a complement to this theorem, we characterize forms $Q_{1} \sim Q_{2}$ with the same fundamental discriminant $d_{1}=d_{2}$ (Theorem 7.4).

Theorem. Let $Q_{1}=\left\langle a_{1}, b_{1}, c_{1}\right\rangle$ be a form with $\left|D_{1}\right|>4$. Then there exists a form $Q_{2} \sim Q_{1}$ such that $\left|D_{2}\right|>\left|D_{1}\right|$ and $d_{1}=d_{2}=d$ if and only if one of the following holds:

(i) $d \equiv 1(\bmod 8)$ and $2 \nmid D_{1}$;

(ii) $2 \mid D_{1}$ and either $b_{1}=a_{1}$ or $a_{1}=c_{1}$.

These theorems together prove the conjecture of Jagy and Kaplansky in the affirmative regarding pairs that represent the same odd primes. (See also Remark 6.4 at the end of this article.)

We now give an outline of the proof. To a form $Q$, we associate an ideal class in an imaginary quadratic order and, by the Artin map, to this ideal class we associate an element of a ring class group (Proposition 2.4). The representability of a prime $p$ by the form $Q$ then amounts to a certain splitting condition on $p$ in the ring class field associated to $Q$. Therefore, two forms $Q_{1}, Q_{2}$ represent almost the same primes if and only if they give rise to the same splitting data, which can be formally thought of as an open and closed subset $S \subset \operatorname{Gal}(\overline{\mathbb{Q}} / \mathbb{Q})$ (Lemma 3.6). By Galois theory, such a set has a (unique) minimal field of definition $L$ (Proposition 3.5).

We first treat the case when the forms $Q_{1}, Q_{2}$ have different fundamental discriminants $d_{1} \neq d_{2}$. Group theoretic considerations show that $Q_{1}, Q_{2}$ have the same genus class field, contained in the field $L$, and that their ring class groups are of type dividing $(2, \ldots, 2,4)$, i.e., they can be embedded in $(\mathbb{Z} / 2 \mathbb{Z})^{r} \oplus \mathbb{Z} / 4 \mathbb{Z}$ for some $r \in \mathbb{Z}_{\geq 0}$ (Proposition 4.5). We then extend existing methods for bounding class groups of imaginary quadratic fields and, using a computer, effectively determine all possible ring class extensions which may arise from the forms $Q_{1} \sim Q_{2}$ (§5). From this finite data we can then list all possible pairs of quadratic forms which represent almost the same primes $(\S 6)$.

When $Q_{1}, Q_{2}$ have the same fundamental discriminant $d_{1}=d_{2}$, we can by classical methods determine necessary and sufficient conditions for $Q_{1} \sim Q_{2}(\S 7)$.

As a side result which may be of independent interest, we provide the following classification of class groups of quadratic orders (Theorem 8.2).

Proposition. There are at least 226 and at most 227 fundamental discriminants $D=d<0$ such that $\mathrm{Cl}(d)$ is of type dividing $(2, \ldots, 2,4)$, and there are at least 199 and at most 205 such discriminants $D$ of nonmaximal orders.

These orders are listed in Tables $7-16$ at the end of this article.

\section{RING CLASS FIELDS}

In this section, we fix notation and summarize without proof the few results we will need from class field theory and the theory of $L$-functions (see e.g. Cox, La, and $[\mathrm{Wa}]$ ). 
Let $K=\mathbb{Q}(\sqrt{d})$ be an imaginary quadratic field of discriminant $d<0$ with ring of integers $A$. For an integer $f \geq 1$, consider the order $A_{f}=\mathbb{Z}+f A$; the discriminant of $A_{f}$ is $D=d f^{2}$. There is a bijection between the set $I(A)$ of ideals of $A$ coprime to $f$ and the set $I\left(A_{f}\right)$ of ideals of $A_{f}$ coprime to $f$, given by $\mathfrak{a} \mapsto \mathfrak{a} \cap A_{f}$ and conversely $\mathfrak{a}_{f} \mapsto \mathfrak{a}_{f} A$. Let $\mathrm{Cl}_{f}(d)=\mathrm{Cl}(D)=\operatorname{Pic}\left(A_{f}\right)$ be the class group of the order $A_{f}$, namely the group of invertible $A_{f}$-ideals modulo principal $A_{f}$-ideals. Given an ideal $\mathfrak{a} \subset A$ prime to $f$, the $A_{f}$-module $\mathfrak{a} \cap A_{f}$ is trivial in $\mathrm{Cl}(D)$ if and only if $\mathfrak{a}$ is principal and generated by an element $\alpha$ with $\alpha \equiv z(\bmod f A)$ for some $z \in \mathbb{Z}$. We write $h_{f}(d)=h(D)=\# \operatorname{Cl}(D)$.

Proposition 2.1 ([Cox, $\S 9])$. There is a unique field $R_{(f)} \supset K$ inside $\bar{K}$ that is abelian over $K$ with the following properties:

(i) Each prime $\mathfrak{p}$ of $K$ coprime to $f$ is unramified in $R_{(f)}$.

(ii) There is an isomorphism

$$
\begin{aligned}
\mathrm{Cl}_{f}(d) & \cong \operatorname{Gal}\left(R_{(f)} / K\right), \\
{\left[\mathfrak{p} \cap A_{f}\right] } & \mapsto \operatorname{Frob}_{\mathfrak{p}}
\end{aligned}
$$

for each prime $\mathfrak{p}$ of $K$ coprime to $f$.

The field $R_{(f)}$ is the largest abelian extension of $K$ of conductor dividing $(f)$ in which all but finitely many primes of $K$ inert over $\mathbb{Q}$ split completely.

The exact sequence

$$
1 \rightarrow \operatorname{Gal}\left(R_{(f)} / K\right) \rightarrow \operatorname{Gal}\left(R_{(f)} / \mathbb{Q}\right) \rightarrow \operatorname{Gal}(K / \mathbb{Q}) \rightarrow 1
$$

splits, and a choice of splitting gives an isomorphism

$$
\operatorname{Gal}\left(R_{(f)} / \mathbb{Q}\right) \cong \operatorname{Gal}\left(R_{(f)} / K\right) \rtimes \operatorname{Gal}(K / \mathbb{Q})
$$

where the nontrivial element of $\operatorname{Gal}(K / \mathbb{Q}) \cong \mathbb{Z} / 2 \mathbb{Z}$ acts on $\operatorname{Gal}\left(R_{(f)} / K\right)$ by inversion $\sigma \mapsto \sigma^{-1}$.

The field $R_{(f)}$ is called the ring class field of $K$ of modulus $f$, and the map $\mathrm{Cl}_{f}(d) \cong \operatorname{Gal}\left(R_{(f)} / K\right)$ is known as the Artin isomorphism.

Remark 2.2. As $\operatorname{Gal}\left(R_{(f)} / K\right)$ is abelian, we see from the proposition that the conjugacy class of an element $\sigma \in \operatorname{Gal}\left(R_{(f)} / K\right)$ in $\operatorname{Gal}\left(R_{(f)} / \mathbb{Q}\right)$ is equal to $\left\{\sigma, \sigma^{-1}\right\}$.

Corollary 2.3. Let $f_{1}, f_{2} \in \mathbb{Z}_{\geq 1}$, and let $f=\operatorname{gcd}\left(f_{1}, f_{2}\right)$. Then $R_{\left(f_{1}\right)} \cap R_{\left(f_{2}\right)}=$ $R_{(f)}$.

Proof. The conductor of $R_{\left(f_{1}\right)} \cap R_{\left(f_{2}\right)}$ divides both $\left(f_{1}\right)$ and $\left(f_{2}\right)$; therefore it divides $(f)$ and has all but finitely many primes of $K$ inert over $\mathbb{Q}$ split completely, hence $R_{\left(f_{1}\right)} \cap R_{\left(f_{2}\right)} \subset R_{(f)}$. Note also that $R_{(f)} \subset R_{\left(f_{1}\right)} \cap R_{\left(f_{2}\right)}$ since $f \mid f_{1}$ and $f \mid f_{2}$; therefore equality holds.

Proposition 2.4 ([Cox, Theorem 7.7]). Let $D=d f^{2}<0$ be a discriminant. Then there is a bijection between the set of $S L_{2}(\mathbb{Z})$-reduced forms of discriminant $D$ and the set of ideal classes in $\mathrm{Cl}(D)$ by the identifications

$$
Q=\langle a, b, c\rangle=a x^{2}+b x y+c y^{2} \longleftrightarrow[\mathfrak{a}]=[(a,(-b+f \sqrt{d}) / 2)] .
$$

Let $Q$ be a form, with $Q \leftrightarrow[\mathfrak{a}]$ for $\mathfrak{a}$ an ideal of $A$ and $[\mathfrak{a}]$ associated to $\sigma \in$ $\operatorname{Gal}\left(R_{(f)} / K\right)$ under the Artin map. Let $p \nmid f$ be prime. Then $p$ is represented by $Q$ if and only if $[\mathfrak{a}]$ contains an integral ideal of norm $p$, which holds if and only if we have $\operatorname{Frob}_{p}=\left\{\sigma, \sigma^{-1}\right\} \subset \operatorname{Gal}\left(R_{(f)} / \mathbb{Q}\right)$. 
Remark 2.5. When considering primes represented by a form, we naturally link a Frobenius element together with its inverse; note that exactly one element of any conjugacy class $\left\{\sigma, \sigma^{-1}\right\}$ is associated with a $G L_{2}(\mathbb{Z})$-reduced form.

Remark 2.6. Since $h_{f}(d)=\left[R_{(f)}: K\right]$, it follows from the Chebotarev density theorem that the density of the set of primes represented by $Q$ is equal to $1 /\left(2 h_{f}(d)\right)$ if the corresponding element $\sigma$ has order $\leq 2$ (i.e., $\sigma=\sigma^{-1}$ ) and $1 / h_{f}(d)$ otherwise.

Lemma 2.7. The forms $Q_{1}, Q_{2}$ represent almost the same primes $\left(Q_{1} \sim Q_{2}\right)$ if and only if for almost all primes $p$ of $\mathbb{Q}$, we have

$$
\operatorname{Frob}_{p}=\left\{\sigma_{1}, \sigma_{1}^{-1}\right\} \subset \operatorname{Gal}\left(R_{1} / \mathbb{Q}\right) \Longleftrightarrow \operatorname{Frob}_{p}=\left\{\sigma_{2}, \sigma_{2}^{-1}\right\} \subset \operatorname{Gal}\left(R_{2} / \mathbb{Q}\right) .
$$

Remark 2.8. It follows from this that if $Q_{1}, Q_{2}$ are forms with the same discriminant $D_{1}=D_{2}$, then $Q_{1} \sim Q_{2}$ if and only if $Q_{1}=Q_{2}$.

Proposition 2.9. The field $P_{(f)} \subset R_{(f)}$ given by

$$
\operatorname{Gal}\left(P_{(f)} / K\right) \cong \mathrm{Cl}_{f}(d) / \mathrm{Cl}_{f}(d)^{2}
$$

is the largest subextension of $R_{(f)} \supset K$ with Galois group $\operatorname{Gal}\left(R_{(f)} / K\right)$ of exponent dividing 2. Moreover, the extension $P_{(f)} \supset \mathbb{Q}$ is itself abelian and of exponent 2 , and is the largest abelian subextension of $R_{(f)} \supset \mathbb{Q}$.

The field $P_{(f)}$ is called the genus class field of $K$ of modulus $f$.

Proof. This follows immediately from Proposition 2.1, as inversion acts trivially on a group of exponent dividing 2 .

We can compute the genus class field explicitly as follows. For $p$ an odd prime we write $p^{*}=(-1)^{(p-1) / 2} p$.

Corollary 2.10. Let $p_{1}, \ldots, p_{r}$ be the odd primes dividing $D$ and let

$$
K^{*}=K\left(\sqrt{p_{1}^{*}}, \ldots, \sqrt{p_{r}^{*}}\right) .
$$

Then the genus class field $P_{(f)}$ of $K$ is as follows:

$$
P_{(f)}= \begin{cases}K^{*}(\sqrt{-1}), & \text { if } d \equiv 1(\bmod 4) \text { and } 4 \| f, \\ K^{*}(\sqrt{-1}, \sqrt{2}), & \text { if } d \equiv 1(\bmod 4) \text { and } 8 \mid f, \\ K^{*}(\sqrt{2}), & \text { if } d \equiv 4(\bmod 8) \text { and } 4 \mid f, \\ K^{*}(\sqrt{-1}), & \text { if } d \equiv 0(\bmod 8) \text { and } 2 \mid f, \\ K^{*}, & \text { otherwise. }\end{cases}
$$

Proof. See [Cox, $\S 6 \mathrm{~A}]$ for the case $f=1$. The case $f>1$ is a standard calculation and follows in a similar way.

Corollary 2.11. The odd primes $p$ which ramify in $P_{(f)}$ are exactly the odd primes that divide $D$.

If $G$ is an abelian group and $n \in \mathbb{Z}_{>0}$, then we define $G[n]=\{g \in G: n g=0\}$.

Corollary 2.12. If $d$ has $g$ distinct prime factors, then $\mathrm{Cl}(d)[2] \cong(\mathbb{Z} / 2 \mathbb{Z})^{g-1}$.

For a fundamental discriminant $d<0$, let

$$
\chi(n)=\chi_{d}(n)=\left(\frac{d}{n}\right)
$$

denote the Kronecker symbol. 
Lemma 2.13 ([Cox, Theorem 7.24]). The sequence

$$
1 \rightarrow A_{f}^{*} \rightarrow A^{*} \rightarrow(A / f A)^{*} /(\mathbb{Z} / f \mathbb{Z})^{*} \rightarrow \mathrm{Cl}_{f}(d) \rightarrow \mathrm{Cl}(d) \rightarrow 1,
$$

is exact, and

$$
h(D)=\frac{h(d) f}{\left[A^{*}: A_{f}^{*}\right]} \prod_{p \mid f}\left(1-\left(\frac{d}{p}\right) \frac{1}{p}\right) .
$$

In the sequel, we will use lower bounds on the sizes of the class groups of quadratic fields. If we write

$$
L(s, \chi)=\sum_{n=1}^{\infty} \frac{\chi(n)}{n^{s}}=\sum_{n=1}^{\infty} \frac{(d / n)}{n^{s}}
$$

for $s \in \mathbb{C}$ with $\operatorname{Re}(s)>0$, then

$$
h(d)=\frac{\sqrt{|d|}}{\pi} L(1, \chi)
$$

for $|d|>4$ (see e.g. [D, §6]). By the Brauer-Siegel theorem, $\log h(d)$ is asymptotic to $\log (\sqrt{|d|})$ as $|d| \rightarrow \infty$; by a result of Siegel $[\underline{\underline{S}}$, we know that for every $\epsilon>0$, there exists a constant $c(\epsilon)$ such that

$$
L(1, \chi)>\frac{c(\epsilon)}{|d|^{\epsilon}} ;
$$

however, this constant $c(\epsilon)$ is not known to be effectively computable. Therefore we will use the following result on the size of $L(1, \chi)$.

Lemma 2.14 (Tatuzawa T]). For any $0<\epsilon<1 / 2$, there is at most one fundamental discriminant $d<0$ with $\log |d|>\max (1 / \epsilon, 11.2)$ satisfying

$$
L(1, \chi) \leq 0.655 \frac{\epsilon}{|d|^{\epsilon}} .
$$

\section{Fields Of DEFInition}

We now proceed with a bit of Galois theory. The reader may prefer on a first reading to skip to the next section and refer back when needed.

Let $K$ be a field with separable closure $\bar{K}$ and absolute Galois group $G=$ $\operatorname{Gal}(\bar{K} / K)$, equipped with the Krull topology. Let $E$ be a finite extension of $K$ contained in $\bar{K}$ and let $\operatorname{Hom}_{K}(E, \bar{K})$ denote the set of $K$-embeddings $E \hookrightarrow \bar{K}$; if $E$ is Galois over $K$, then $\operatorname{Hom}_{K}(E, \bar{K})$ is identified with $\operatorname{Gal}(E / K)$. We have a restriction map

$$
\begin{aligned}
\operatorname{res}_{E}: G & \rightarrow \operatorname{Hom}_{K}(E, \bar{K}) \\
\sigma & \mapsto \operatorname{res}_{E}(\sigma)=\left.\sigma\right|_{E} .
\end{aligned}
$$

The map res $\operatorname{ris}_{E}$ is continuous if the finite set $\operatorname{Hom}_{K}(E, \bar{K})$ is equipped with the discrete topology.

Lemma 3.1. A subset $S \subset G$ is open and closed if and only if there exist a finite extension $L \supset K$ contained in $\bar{K}$ and a set $T \subset \operatorname{Hom}_{K}(L, \bar{K})$ such that $S=\operatorname{res}_{L}^{-1}(T)$. 
Proof. Given $T \subset \operatorname{Hom}_{K}(L, \bar{K})$, note that $T$ is open and closed (in the discrete topology) and $\operatorname{res}_{L}$ is a continuous map.

Conversely, suppose $S \subset G$ is open and closed. Then for every $\sigma \in S$, there exists an open neighborhood $U_{\sigma}=\operatorname{res}_{E_{\sigma}}^{-1}\left(\left.\sigma\right|_{E_{\sigma}}\right) \subset S$ of $\sigma$ given by some finite extension $E_{\sigma} \supset K$. Together these give an open cover $\left\{U_{\sigma}\right\}_{\sigma \in S}$ of $S$. Since $G$ is compact and $S$ is closed, $S$ is itself compact and therefore is covered by $\left\{U_{\sigma}\right\}_{\sigma \in S^{\prime}}$ for $S^{\prime} \subset S$ a finite subset. Let $L$ be the compositum of the fields $E_{\sigma}$ for $\sigma \in S^{\prime}$, and let

$$
T=\left\{\tau \in \operatorname{Hom}_{K}(L, \bar{K}):\left.\tau\right|_{E_{\sigma}}=\left.\sigma\right|_{E_{\sigma}} \text { for some } \sigma \in S^{\prime}\right\} .
$$

Then by construction $S=\operatorname{res}_{L}^{-1}(T)$.

Definition 3.2. Given an open and closed set $S \subset G$, we say that $L$ is a field of definition for $S$ if $L \supset K$ is a finite extension and there is a subset $T \subset \operatorname{Hom}_{K}(L, \bar{K})$ such that $S=\operatorname{res}_{L}^{-1}(T)$.

Remark 3.3. If $L$ is a field of definition with $S=\operatorname{res}_{L}^{-1}(T)$ for some subset $T \subset$ $\operatorname{Hom}_{K}(L, \bar{K})$, then in fact $T=\left.S\right|_{L}$. Therefore $L$ is a field of definition for $S$ if and only if $\operatorname{res}_{L}^{-1}\left(\left.S\right|_{L}\right)=S$, i.e., for every $\sigma \in G$ and $\tau \in S$ such that $\left.\sigma\right|_{L}=\left.\tau\right|_{L}$ we have $\sigma \in S$. It follows immediately from this that if $L$ is a field of definition for $S$ and $M \supset L$ is a finite extension, then $M$ is also a field of definition for $S$.

Put in these terms, Lemma 3.1 states that every open and closed subset $S \subset G$ has a field of definition.

Definition 3.4. A field of definition $L$ for $S$ is minimal if for every field of definition $E$ for $S$, we have $L \subset E$.

If a minimal field of definition $L$ exists, it is obviously unique.

Proposition 3.5. For any open and closed set $S \subset G$, there exists a minimal field of definition $L(S)$ for $S$.

Proof. Consider the set

$$
H(S)=\{\sigma \in G: S \sigma=S\} \subset G ;
$$

we claim that $L(S)=\bar{K}^{H(S)}$.

The set $H(S)$ is clearly a subgroup of $G$. Let $L \supset K$ be a finite extension with $H=\operatorname{Gal}(\bar{K} / L)$. Then by Remark 3.3. the field $L$ is a field of definition for $S$ if and only if the following statement holds:

$$
\text { For all } \sigma \in G \text { and } \tau \in S \text {, if }\left.\sigma\right|_{L}=\left.\tau\right|_{L} \text {, then } \sigma \in S \text {. }
$$

Note $\left.\sigma\right|_{L}=\left.\tau\right|_{L}$ if and only if $\tau^{-1} \sigma \in H$, therefore $L$ is a field of definition if and only if for all $\tau \in S$, we have $\tau H \subset S$, which holds if and only if $S H=S$, i.e., $H \subset H(S)$, or equivalently $L \supset \bar{K}^{H(S)}=L(S)$. Since a field of definition for $S$ exists by Lemma 3.1 we see that $L(S)$ is a finite extension of $K$. Therefore $L(S)$ is the minimal field of definition for $S$.

We now relate this notion to representation of primes. Let $K$ be a number field. Let $\Pi$ be the set of equivalence classes of sets of primes of $K$, where two sets are equivalent if they differ only by a finite set. To every open and closed set $S \subset G$ which is closed under conjugation, we can associate a set $\mathcal{P}(S)$ of primes of $K$ : namely, if $L$ is a field of definition for $S$, we associate the set

$$
\mathcal{P}(S)=\left\{\mathfrak{p} \text { a prime of } K: \mathfrak{p} \nmid \operatorname{disc}(L / K),\left.\operatorname{Frob}_{\mathfrak{p}} \subset S\right|_{L}\right\},
$$


where Frob $_{\mathfrak{p}}$ is the Frobenius class at the prime $\mathfrak{p}$. If $M$ is another field of definition for $S$, then the two sets given by $L$ and $M$ differ by only a finite set, contained in the set of primes that ramify in $L$ or in $M$, and hence we have a well-defined element $\mathcal{P}(S) \in \Pi$.

Lemma 3.6. The above association $S \mapsto \mathcal{P}(S)$ is injective. The minimal field of definition for $S$ is Galois over K.

Proof. Suppose that $S \neq S^{\prime}$. By Remark 3.3, the compositum of a field of definition for $S$ and for $S^{\prime}$ is a field of definition for both. Therefore there exists a common field of definition $L$ for $S, S^{\prime}$ which by the same remark we may take to be Galois over $K$, hence $\left.S\right|_{L} \neq\left. S^{\prime}\right|_{L}$. Suppose then that $\left.\left.\sigma \in S\right|_{L} \backslash S^{\prime}\right|_{L}$; by the Chebotarev density theorem [La, p. 169], there exist infinitely many primes $\mathfrak{p}$ of $K$ such that Frob $_{\mathfrak{p}}$ is equal to the conjugacy class of $\sigma$, which is disjoint from $\left.S^{\prime}\right|_{L}$ since $S^{\prime}$ is closed under conjugation. Therefore $\mathcal{P}(S) \neq \mathcal{P}\left(S^{\prime}\right)$.

For the second statement, let $S$ be a set with minimal field of definition $L$ and let $\alpha \in G$. Then the set $\alpha S \alpha^{-1}$ has minimal field of definition $\alpha L$ : we have $\left.\alpha \sigma \alpha^{-1}\right|_{\alpha L}=\left.\alpha \tau \alpha^{-1}\right|_{\alpha L}$ if and only if $\left.\sigma\right|_{L}=\left.\tau\right|_{L}$. Therefore if $S$ is closed under conjugation, then $\alpha L=L$ and the minimal field of definition is Galois over $K$.

\section{Characterizing equivalence via Class Groups}

In this section, we characterize the class groups which can arise from a pair of quadratic forms which represent almost the same primes. In particular (Proposition 4.5), if the forms have different fundamental discriminants, we show that they must either be of exponent dividing 2 or of type $(2, \ldots, 2,4)$. This proposition allows us to give necessary and sufficient conditions for the existence of such pairs with different fundamental discriminants (Theorem 4.7) and the same fundamental discriminant (Proposition 4.8).

Throughout the following sections, we will utilize the following notation.

Notation 4.1. Let $Q$ denote a (primitive, $G L_{2}(\mathbb{Z})$-reduced, integral positive definite binary quadratic) form of discriminant $D=d f^{2}$, where $d<0$ is the fundamental discriminant. Let $K=\mathbb{Q}(\sqrt{D})=\mathbb{Q}(\sqrt{d})$, and let $R$ be the ring class field of $K$ of modulus $f$ with $h(D)=\# \mathrm{Cl}(D)=[R: K]$ and genus class field $P \supset K$. By Proposition [2.1, the form $Q$ corresponds to an ideal class $[\mathfrak{a}]$ and to an element $\sigma \in \operatorname{Gal}(R / K)$. We define the set

$$
S=\operatorname{res}_{R}^{-1}\left(\left\{\sigma, \sigma^{-1}\right\}\right) \subset \operatorname{Gal}(\overline{\mathbb{Q}} / \mathbb{Q}) .
$$

Note that $\mathcal{P}(S)$ (as in Lemma 3.6) is the set of primes represented by $Q$, up to a finite set (contained in the set of primes dividing $f$ ).

The set $S$ is open and closed in $\operatorname{Gal}(\overline{\mathbb{Q}} / \mathbb{Q})$ and closed under conjugation. Let $L=L(S)$ be the minimal field of definition for $S$, which exists by Proposition 3.5. since $R$ is a field of definition for $S$, we have $L \subset R$. (Note here we take the base field in $\S 2$ to be $\mathbb{Q}$.)

Lemma 4.2. We have $[R: L] \leq 2$, and $[R: L]=2$ if and only if $\left.\sigma\right|_{L}$ has order 2 and $\sigma$ has order 4 . Moreover, we have $P \subset L$. 
Proof. Since $\left.S\right|_{R}=\left\{\sigma, \sigma^{-1}\right\}$, we have

$$
2 \geq\left. \# S\right|_{R}=[R: L]\left(\left.\# S\right|_{L}\right)
$$

so $[R: L] \leq 2$. Moreover, $[R: L]=2$ if and only if $\left.\# S\right|_{R}=2$ and $\left.\# S\right|_{L}=1$, which holds if and only if $\left.\sigma\right|_{L}=\left.\sigma^{-1}\right|_{L}$ and $\sigma \neq \sigma^{-1}$, i.e., $\left.\sigma\right|_{L}$ has order 2 and $\sigma$ has order 4 .

To prove that $P \subset L$, note that in either case $\operatorname{Gal}(R / L)$ is generated by $\sigma^{2} \in$ $\mathrm{Cl}_{f}(d)^{2}=\operatorname{Gal}(R / P)$.

Now suppose that $Q_{1}$ and $Q_{2}$ are a pair of forms, following Notation 4.1 with appropriate subscripts. It is immediate from Lemma 2.7 that $Q_{1}$ and $Q_{2}$ have the same set $\mathcal{P}(S)$ (up to a finite set) and by the injectivity of Lemma 3.6 the same set $S$, hence the same minimal field of definition $L$.

Lemma 4.3. If $Q_{1} \sim Q_{2}$, then we have $K_{1} K_{2} \subset L$, and $K_{1} K_{2}$ is fixed by all elements of $S$. Moreover, we have equality of genus class fields $P_{1}=P_{2}$.

Proof. This follows immediately from the fact that $K_{i} \subset P_{i} \subset L$ and that $P_{i}$ is the maximal subextension of $L / \mathbb{Q}$ of exponent dividing 2 .

We denote this common genus class field by $P=P_{1}=P_{2}$.

Corollary 4.4. If $Q_{1} \sim Q_{2}$, then $\left.\sigma_{1}\right|_{P}=\left.\sigma_{2}\right|_{P}$.

Proof. Note $\left.\sigma_{2}\right|_{P}=\left.\sigma_{2}^{-1}\right|_{P}$. Since $P \subset L$, by Lemma 2.7 we conclude $\left.\sigma_{1}\right|_{P}=$ $\left.\sigma_{2}\right|_{P}$.

We now distinguish two cases, depending on whether $Q_{1}, Q_{2}$ have the same fundamental discriminant.

Proposition 4.5. Suppose $Q_{1} \sim Q_{2}$ and $K_{1} \neq K_{2}$. Then for $i=1,2$, the group $\operatorname{Gal}\left(R_{i} / K_{i}\right)$ is of type dividing $(2, \ldots, 2,4)$, and the minimal field of definition is equal to the common genus class field, i.e., $L=P$.

Proof. Let $\alpha \in \operatorname{Gal}(L / \mathbb{Q})$ be any element of order not dividing 2. From Proposition 2.1 we have

$$
\operatorname{Gal}(L / \mathbb{Q})=\operatorname{Gal}\left(L / K_{i}\right) \rtimes \operatorname{Gal}\left(K_{i} / \mathbb{Q}\right)
$$

where the nontrivial element of $\operatorname{Gal}\left(K_{i} / \mathbb{Q}\right) \cong \mathbb{Z} / 2 \mathbb{Z}$ acts on $\operatorname{Gal}\left(L / K_{i}\right)$ by inversion. Suppose that $\alpha \in \operatorname{Gal}(L / \mathbb{Q})$ is an element of order $>2$. Then in fact $\alpha \in \operatorname{Gal}\left(L / K_{i}\right)$, since every element of $\operatorname{Gal}(L / \mathbb{Q}) \backslash \operatorname{Gal}\left(L / K_{i}\right)$ has order 2. Therefore the centralizer of $\alpha$ in $\operatorname{Gal}(L / \mathbb{Q})$ is the group $\operatorname{Gal}\left(L / K_{i}\right)$. Hence if such an $\alpha$ exists, then $K_{i}$ is determined by $L$, so $K_{1}=K_{2}$. So $K_{1} \neq K_{2}$ implies that $\operatorname{Gal}(L / \mathbb{Q})$ is of exponent 2 , and then from the exact sequence

$$
0 \rightarrow \operatorname{Gal}\left(R_{i} / L\right) \rightarrow \operatorname{Gal}\left(R_{i} / K_{i}\right) \rightarrow \operatorname{Gal}\left(L / K_{i}\right) \rightarrow 0
$$

and the fact that $\left[R_{i}: L\right] \leq 2$ we see that $\operatorname{Gal}\left(R_{i} / K_{i}\right)$ is of type dividing $(2, \ldots, 2,4)$.

The second statement then follows, since then $L \subset P$.

Remark 4.6. This proposition answers a question of Jagy and Kaplansky [JK]. Two ideal classes are said to be in the same genus if their ratio is a square of an ideal class. Jagy and Kaplansky call a form $Q$ bi-idoneal if its genus consists of only $Q$ and its inverse; in their terminology, every "non-trivial" pair of forms (i.e., $d_{1} \neq d_{2}$ ) representing the same primes they found was bi-idoneal. 
Proposition 4.5 shows that this always holds: if $Q_{1}, Q_{2}$ represent the same primes outside a finite set and $d_{1} \neq d_{2}$, then $Q_{1}$ and $Q_{2}$ are bi-idoneal. This follows from the fact that a finite abelian group $G$ has $\#\left(G^{2}\right) \leq 2$ if and only if $G$ is of type dividing $(2, \ldots, 2,4)$.

We can now formulate necessary and sufficient conditions for the existence of pairs which represent almost the same primes with different fundamental discriminants.

Theorem 4.7. Let $Q_{1}, Q_{2}$ be forms, and suppose that $K_{1} \neq K_{2}$. Then $Q_{1} \sim Q_{2}$ if and only if both of the following hold:

(i) $R_{1}$ and $R_{2}$ have the same genus class field $P$, and

$$
\left.\sigma_{1}\right|_{P}=\left.\sigma_{2}\right|_{P} \in \operatorname{Gal}\left(P / K_{1} K_{2}\right) .
$$

(ii) For $i=1,2$, the group $\operatorname{Gal}\left(R_{i} / K_{i}\right)$ is either of exponent dividing 2 , or is of type $(2, \ldots, 2,4)$ and $\sigma_{i}$ has order 4 .

Proof. We have shown these conditions are necessary: condition (i) follows from Lemma 4.3 and Corollary 4.4, and (ii) follows from Proposition 4.5.

Now we show that these conditions are also sufficient. For $i=1,2$, let $L_{i}$ be the minimal field of definition of $S_{i}$ (as in Notation 4.1, with subscripts). From Lemma 4.2 and (i), we have $P \subset L_{i}$, and since $R_{i}$ is a field of definition for $S_{i}$ we have $L_{i} \subset R_{i}$. We will now show that in fact $L_{i}=P$. From (ii), either $\operatorname{Gal}\left(R_{i} / K_{i}\right)$ is of exponent dividing 2 and $R_{i}=L_{i}=P$ already, or $\operatorname{Gal}\left(R_{i} / K_{i}\right)$ is of type $(2, \ldots, 2,4)$ and $\sigma_{i}$ has order 4 . But then $P$ is a field of definition for $S_{i}$, since $\operatorname{res}_{R}^{-1}\left(\left.\sigma_{i}\right|_{P}\right)=\left\{\sigma_{i}, \sigma_{i}^{-1}\right\}$, hence $L_{i} \subset P$, so $L_{i}=P$ in this case as well. Therefore $L_{1}=L_{2}=L$.

Now let $p$ be a prime which is unramified in $R_{1} R_{2}$. Then $\sigma_{1} \in$ Frob $\left._{p}\right|_{L}$ if and only if $\left.\sigma_{2} \in \operatorname{Frob}_{p}\right|_{L}$, so then $Q_{1} \sim Q_{2}$ by Lemma 2.7

To conclude this section, we consider the case when two forms have the same fundamental discriminant.

Proposition 4.8. Let $Q_{1}, Q_{2}$ be forms with $d_{1}=d_{2}=d$.

Suppose that $f_{1} \mid f_{2}$, and let

$$
\phi: \mathrm{Cl}\left(D_{2}\right) \rightarrow \mathrm{Cl}\left(D_{1}\right)
$$

be the natural (restriction) map. Then $Q_{1} \sim Q_{2}$ if and only if $\phi\left(\sigma_{2}\right)=\sigma_{1}$ and one of the following holds: either $\phi$ is an isomorphism, or

More generally, we have $Q_{1} \sim Q_{2}$ if and only if there exists a form $Q$ of discriminant $D=d f^{2}$ with $Q_{1} \sim Q \sim Q_{2}$, where $f=\operatorname{gcd}\left(f_{1}, f_{2}\right)$.

Proof. From Proposition 2.1, we conclude that $R_{1} \subset R_{2}$. If $R_{1}=R_{2}$, then $\phi$ is an isomorphism. Otherwise, by Lemma [4.2, we have $\left[R_{2}: R_{1}\right]=2$ and $Q_{1} \sim Q_{2}$ if and only if $\operatorname{res}_{R_{2}}^{-1}\left(\sigma_{1}\right)=\left\{\sigma_{2}, \sigma_{2}^{-1}\right\}$, where $\sigma_{2}$ has order 4 and $\sigma_{1}$ has order 2 . Now $\sigma_{1}$ has order 2 if and only if $\sigma_{2}^{2} \in \operatorname{ker} \phi$, and $\operatorname{ker} \phi$ is generated by $\sigma_{2}^{2}$ if and only if $\sigma_{2}$ has order 4 , which is condition $(\dagger)$. This proves the first statement.

To prove the second statement, let $R=R_{f}$. Then by Corollary $2.3, R_{1} \cap R_{2}=R$. Since $L \subset R_{1}, R_{2}$ we see that $L \subset R$; therefore by Remark 3.3 the field $R$ is a field of definition for $S$. Let $Q$ be the form of discriminant $d f^{2}$ associated to $\left.\sigma_{1}\right|_{R}$. Again 
by Lemma 4.2 we see that either $R_{1}=R$, in which case $Q_{1} \sim Q$, or $\left[R_{1}: R\right]=2$, in which case $L=R$ and as above we have $Q_{1} \sim Q$. Similarly, let $Q^{\prime}$ be the form of discriminant $d f^{2}$ associated to $\left.\sigma_{2}\right|_{R}$. Then $Q_{2} \sim Q^{\prime}$. Since $Q_{1} \sim Q_{2}$, we have $Q \sim Q^{\prime}$. But $Q$ and $Q^{\prime}$ have the same discriminant, which implies that $Q=Q^{\prime}$, by Remark 2.8

\section{BOUNDING CLASS GROUPS}

Recall as in the introduction, to every equivalence class $C$ of forms, we associate the set $\delta(C)$ of fundamental discriminants of the forms in $C$ as well as the set $\Delta(C)$ of discriminants of forms in $C$. In this section, we will prove that there are only finitely many equivalence classes $C$ with $\# \delta(C) \geq 2$. More precisely, we will prove the following statement.

Proposition 5.1. The sets

$$
\mathcal{D}_{\delta}=\bigcup_{\# \delta(C) \geq 2} \delta(C) \text { and } \mathcal{D}_{\Delta}=\bigcup_{\# \delta(C) \geq 2} \Delta(C),
$$

are finite and effectively computable. Moreover, $\# \mathcal{D}_{\delta} \leq 226$ and $\# \mathcal{D}_{\Delta} \leq 425$.

First note the following lemma.

Lemma 5.2 ([We, Lemma 5$])$. Let $K=\mathbb{Q}(\sqrt{d})$ have discriminant $d<0$, let $\mathfrak{a}$ be an integral ideal of $K=\mathbb{Q}(\sqrt{d})$ and let $c$ be a positive integer such that $\mathfrak{a}^{c}$ is principal. If $\mathfrak{a}$ is not a principal ideal generated by a rational integer and $\mathfrak{a}$ is prime to $d$, then $(N \mathfrak{a})^{c}>|d| / 4$.

To prove this lemma, one shows that if $(\alpha)=\mathfrak{a}^{c}$, then $\alpha$ is not a rational integer by considering the factorization of $\mathfrak{a}$ in $K$, and therefore $N\left(\mathfrak{a}^{c}\right)=N(\alpha)^{c}>|d| / 4$.

Corollary 5.3. If $\mathrm{Cl}(d)$ has exponent $c$, then for all primes $p$ such that $p^{c} \leq d / 4$ we have $(d / p) \neq 1$.

Proof. Suppose that $(d / p)=1$; then $(p)=\mathfrak{p} \overline{\mathfrak{p}}$ in the ring of integers $A$ of $K=$ $\mathbb{Q}(\sqrt{d})$. Since $N \mathfrak{p}=p$ is not a square, we know that $\mathfrak{p}$ is not generated by a rational integer. The lemma then implies that $(N \mathfrak{p})^{c}=p^{c}>d / 4$.

Lemma 5.4. If $\mathrm{Cl}_{f}(d)$ is of type dividing $(2, \ldots, 2,4)$ and $|d|>2500$, then $f \in$ $\{1,2,3,4,6,8,12\}$.

Proof. Recall the exact sequence of Lemma 2.13 .

$$
1 \rightarrow(A / f A)^{*} /(\mathbb{Z} / f \mathbb{Z})^{*} \rightarrow \mathrm{Cl}_{f}(d) \rightarrow \mathrm{Cl}(d) \rightarrow 1,
$$

where we note that $|d|>4$ implies $A_{f}^{*}=A^{*}$.

Since the map $\mathrm{Cl}_{f}(d) \rightarrow \mathrm{Cl}(d)$ is surjective, we see that $\mathrm{Cl}(d)$ is itself of type dividing $(2, \ldots, 2,4)$. Let $p$ be an odd prime such that $p \mid f$. From Proposition A.1 we conclude that $p^{2} \nmid f$ and $p=3$ or $p=5$. When $|d|>2500$, or equivalently when $|d / 4|>5^{4}$, we cannot have $5 \mid f$, for this can happen only if $\left(d_{i} / 5\right)=1$, which contradicts Corollary 5.3 . If $2 \mid f$, then since $(d / 2)=1$ cannot occur, and $(d / 2)=-1$ implies $3 \mid \mathrm{Cl}_{f}(d)$, we must have $(d / 2)=0$. But then again from the proposition we see that $16 \nmid f$ and $24 \nmid f$.

Let $Q_{1}, Q_{2}$ be forms with $d_{1} \neq d_{2}$. Let $K_{0}$ be the real quadratic field contained in $K_{1} K_{2}$. 
Lemma 5.5. Let $Q_{1} \sim Q_{2}$ and suppose $\left|d_{\min }\right|=\min \left\{\left|d_{1}\right|,\left|d_{2}\right|\right\}>2500$. Then

$$
K_{0} \in\{\mathbb{Q}(\sqrt{2}), \mathbb{Q}(\sqrt{3}), \mathbb{Q}(\sqrt{6})\} .
$$

Moreover, if $p^{4} \leq\left|d_{\min }\right| / 4$ and $p$ is inert in $K_{0}$, then $p$ ramifies in $K_{1}$ and $K_{2}$.

Proof. By Lemma 4.3, the ring class fields $R_{1}$ and $R_{2}$ have the same genus class field, and by Proposition 4.5, the group $\mathrm{Cl}_{f_{i}}\left(d_{i}\right)$ is of type dividing $(2, \ldots, 2,4)$ for $i=1,2$. By Corollary 2.11, the same set of odd primes divides the discriminants $D_{1}, D_{2}$. Then by Lemma 5.4, we see that $d_{1} / d_{2} \in 2^{\mathbb{Z}} 3^{\mathbb{Z}}$. Therefore the discriminant of $K_{0}$ is supported only at the primes 2 and 3 , and $K_{0}$ is one of the fields listed.

Let $p$ be a prime with $p^{4} \leq d_{\min } / 4$ which is inert in $K_{0}$. We know that $\left(d_{1} / p\right),\left(d_{2} / p\right) \neq 1$, by Corollary [5.3 We cannot have $\left(d_{1} / p\right)=\left(d_{2} / p\right)=-1$, as then $\left(d_{1} d_{2} / p\right)=1$, so $p$ splits in $K_{0}$. Therefore say $\left(d_{1} / p\right)=0$; then $p$ is ramified in $K_{1}$ so $p$ is ramified in $K_{1} K_{2}=K_{0} K_{2}$, so $p$ is ramified in $K_{2}$ as well.

Remark 5.6. This lemma proves that given a fundamental discriminant $d$ with $|d|>$ 2500 , one can explicitly determine all possibilities for fundamental discriminants $d^{\prime}$ of forms $Q^{\prime}$ with $Q^{\prime} \sim Q$.

Lemma 5.7. Let $p_{1}=3, p_{2}=5, \ldots$ be the sequence of odd primes in increasing order, and for each $t \in \mathbb{Z}_{\geq 1}$ let

$$
\widehat{d_{t}}=4 p_{1} \ldots p_{t-1} .
$$

Let $d<-3$ be a fundamental discriminant with $g$ distinct prime factors, and let $t \in \mathbb{Z}_{\geq 1}$. Then

$$
|d| \geq \widehat{d}_{t} p_{t}^{g-t}
$$

Proof. First, we prove that $|d| \geq \widehat{d}_{g}$. If $d \equiv 0(\bmod 4)$, then this is clear. If $d \equiv 1$ $(\bmod 4)$ and $g=1$, then by assumption $|d| \geq 7>4$. If $g \geq 2$, then $p_{g} \geq 5$, and therefore

$$
|d| \geq p_{1} \ldots p_{g} \geq 4 p_{1} \ldots p_{g-1} .
$$

It then follows that $|d| \geq \widehat{d}_{g} \geq \widehat{d}_{t} p_{t}^{g-t}$ for $g \geq t$. But for $g<t$, we also have

$$
|d| \geq \widehat{d}_{g}=\frac{\widehat{d_{t}}}{p_{g+1} \cdots p_{t}} \geq \frac{\widehat{d_{t}}}{p_{t}^{t-g}}
$$

as claimed.

By the preceding two lemmas, we can apply the result of Tatuzawa (Lemma 2.14) to obtain the following.

Proposition 5.8. Let $Q_{1}, Q_{2}$ be forms representing almost the same primes such that $d_{1} \neq d_{2}$. Then we have $\min \left\{\left|d_{1}\right|,\left|d_{2}\right|\right\} \leq B=80604484=4 \cdot 67^{4}$.

Proof. Apply Lemma 2.14 with $\epsilon=1 / \log B$. Note that $\log B>11.2$. Since there is at most one possible exceptional discriminant, we may assume without loss of generality that $d=d_{1}$ is not exceptional, hence

$$
h(d)>\left(\frac{0.655}{\pi}\right) \frac{|d|^{1 / 2-1 / \log B}}{\log B} .
$$

We suppose that $|d|>B$ and derive a contradiction. By Lemma [5.5] every prime $p \leq 67$ which is inert in $K_{0}$ must divide $d$. Let $g$ be the number of distinct prime 
factors of $d$; since \# $\mathrm{Cl}(d)[2]=2^{g-1}$ (Corollary 2.12) and $\mathrm{Cl}(d)$ is of type dividing $(2, \ldots, 2,4)$, we see that $h(d) \leq 2^{g}$.

For $b \in \mathbb{Z}_{>0}$, let

$$
d_{0}(b, q)=\prod_{\substack{2<p \leq b \\(p / q)=-1}} p .
$$

From Lemma 5.5, we have three cases to consider. If $K_{0}=\mathbb{Q}(\sqrt{2})$, then $p$ is inert in $K_{0}$ if and only if $p \equiv 3,5(\bmod 8)$. Therefore

$$
d_{0}(67,8)=\prod_{\substack{p \leq 67 \\ p \equiv 3,5(8)}} p=3 \cdot 5 \cdot \ldots \cdot 61 \cdot 67>2.4 \cdot 10^{16},
$$

and by Lemma 5.5. we have $d_{0}(67,8) \mid d$, so $|d| \geq d_{0}(67,8)$. For $K_{0}=\mathbb{Q}(\sqrt{3})$, the prime $p$ is inert in $K_{0}$ if and only if $p \equiv 5,7(\bmod 12)$, so $d_{0}(67,12)=5 \cdot \ldots \cdot 53 \cdot 67$, and $d_{0}(67,12) \mid d$ so $|d|>6.3 \cdot 10^{13}$. In a similar way, for $K_{0}=\mathbb{Q}(\sqrt{6})$, we obtain $d_{0}(67,24)=7 \cdot 11 \cdot \ldots \cdot 61>2.8 \cdot 10^{13}$.

In any case, we see that $|d|>2.8 \cdot 10^{13}$, and hence

$$
2^{g} \geq h(d)>\left(\frac{0.655}{\pi \log B}\right)\left(2.8 \cdot 10^{13}\right)^{1 / 2-1 / \log B}>10897
$$

so $g \geq 14$.

By Lemma 5.7] we have $|d| \geq \widehat{d}_{14} \cdot 47^{g-14}$, where $\widehat{d}_{14}>2.6 \cdot 10^{17}$. But this implies that

$$
\begin{aligned}
2^{g} \geq h(d) & >\left(\frac{0.655}{\pi \log B}\right) \widehat{d}_{14}^{1 / 2-1 / \log B}\left(47^{1 / 2-1 / \log B}\right)^{g-14} \\
& >226989 \cdot 2^{g-14}
\end{aligned}
$$

which is a contradiction.

Proof of Proposition 5.1. First, by an exhaustive list, we find that there are exactly 226 fundamental discriminants $d$ with $|d| \leq B$ such that $\mathrm{Cl}(d)$ is of type dividing $(2, \ldots, 2,4)$. To speed up this computation, we use Corollary 5.3 to rule out many of these discriminants. This was accomplished in MAGMA. (The code is available from the author by request.) By Proposition 5.8, we have missed at most one possible fundamental discriminant from the set $\mathcal{D}_{d}$.

Next, we show that there are exactly 199 discriminants $D=d f^{2}$ of nonmaximal orders with $|d| \leq B$ such that $\mathrm{Cl}_{f}(d)$ is of type dividing $(2, \ldots, 2,4)$. By Lemma 5.4. we know that $f \in\{2,3,4,6,12\}$. We can use any algorithm which computes class groups (e.g. enumeration) to check these finitely many nonmaximal orders.

Now suppose that $Q_{1}, Q_{2}$ are forms that represent the same primes with $\left|d_{1}\right|<$ $\left|d_{2}\right|$. Then $\left|d_{1}\right| \leq B$, and we must show that $\left|d_{2}\right| \leq B$ as well to have computed $\mathcal{D}_{d}$ and therefore $\mathcal{D}_{D}$ as well. If $\left|d_{1}\right| \leq 2500$, then from the list of discriminants we see that $\left|D_{1}\right| \leq 29568$; since $\mathbb{Q}\left(\sqrt{d_{2}}\right) \subset P_{1}$, we see from Lemma 4.3 that $\left|d_{2}\right| \leq$ $4 \cdot 29568<B$. Otherwise, by Remark [5.6 there are only 3 possibilities for $d_{2}$, and since $\left|d_{1}\right| \leq 10920$, it follows that $\left|d_{2}\right| \leq 12 \cdot 10920 \leq B$ as well, completing the proof. 


\section{FINDING THE PAIRS OF QUADRATIC FORMS}

To conclude, we list all forms with $K_{1} \neq K_{2}$. Using Corollary 2.10, we first compute the genus class field for each of the 425 discriminants found in $\S 5$. We find 86 pairs of discriminants for which the genus class fields are equal.

We now apply Theorem 4.7. If the class group of both discriminants are both of exponent 2, then for every $\sigma \in \operatorname{Gal}\left(R / K_{1} K_{2}\right)$, we obtain a pair corresponding to $\sigma_{i}=\sigma \in \operatorname{Gal}\left(R / K_{i}\right)$. For each $i$ such that $\operatorname{Gal}\left(R_{i} / K_{i}\right)$ has a factor $\mathbb{Z} / 4 \mathbb{Z}$, we proceed as follows: for each $\sigma \in \operatorname{Gal}\left(R_{i} / K_{1} K_{2}\right) \subset \operatorname{Gal}\left(R_{i} / K_{i}\right)$ of order 4 , we compute the fixed field of $\left.\sigma\right|_{P}$ by finding a prime $p \nmid D_{i}$ represented by the form $Q \leftrightarrow \sigma$, and compute (using Legendre symbols) the largest subfield of $P$ in which $p$ splits completely. Then every pair $\sigma_{1}, \sigma_{2}$ with the same fixed subfield (so that $\left.\left.\sigma_{1}\right|_{P}=\left.\sigma_{2}\right|_{P}\right)$ gives rise to a pair of forms.

Example 6.1. The discriminants $D_{1}=-1056=-264 \cdot 2^{2}$ and $D_{2}=-2112=$ $-132 \cdot 4^{2}$ give rise to the common genus class field $P=\mathbb{Q}(i, \sqrt{2}, \sqrt{-3}, \sqrt{-11})$, each with class group of type $(2,2,4)$. The forms of order 4 of discriminant -1056 are

$$
\langle 5,2,53\rangle,\langle 15,12,20\rangle,\langle 7,6,39\rangle,\langle 13,6,21\rangle \text {, }
$$

and those of discriminant -2112 are

$$
\langle 17,8,32\rangle,\langle 21,18,29\rangle,\langle 7,4,76\rangle,\langle 19,4,28\rangle \text {. }
$$

The first form $\langle 5,2,53\rangle$ represents the prime 5 , so we compute the Legendre symbols

$$
(-1 / 5),(2 / 5),(-3 / 5),(-11 / 5) \text {, }
$$

and find the fixed field $\mathbb{Q}(i, \sqrt{6}, \sqrt{-11}) \subset P$. Continuing in this way, we find that only the pair $\langle 7,6,39\rangle$ and $\langle 7,4,76\rangle$ have a common fixed field, namely the field $\mathbb{Q}(\sqrt{2}, \sqrt{-3}, \sqrt{11})$, and this proves that they represent the same primes (those which are congruent to $7,79,127,151,175(\bmod 264))$.

Carrying out this calculation for each of the 86 pairs, and supplementing this list with any pairs arising from the same fundamental discriminant, we obtain the forms listed in Tables 1-5.

Theorem 6.2. There are exactly 67 equivalence classes of forms $C$ such that $\# \delta(C) \geq 2$. There are exactly 6 classes with $\# \delta(C)=3$, and there is no class with $\# \delta(C) \geq 4$.

Definition 6.3. The exceptional set $E$ of a form $Q$ is the set of primes $p$ such that $Q$ represents $p$ and there exists a form $Q^{\prime} \sim Q$ such that $Q^{\prime}$ does not represent $p$.

Remark 6.4. Jagy and Kaplansky JK miss the two pairs

$$
\langle 5,0,6\rangle,\langle 11,4,14\rangle \quad \text { and } \quad\langle 3,0,40\rangle,\langle 27,12,28\rangle
$$

in their "near misses" (those pairs with exceptional set not contained in $\{2\}$ ). Moreover, the form $\langle 4,4,9\rangle$ in their paper should be $\langle 4,4,19\rangle$.

\section{FORMS WITH THE SAME FUNDAMENTAL DISCRIMINANT}

In this section, we treat the case when the forms have the same fundamental discriminant. We will again use Notation 4.1. Throughout, let $Q_{1}, Q_{2}$ be forms with $d_{1}=d_{2}=d<0$.

If $f_{1}=f_{2}$, so that $D_{1}=D_{2}$, then by Remark 2.8 either $Q_{1}=Q_{2}$ or $Q_{1} \nsim Q_{2}$. So without loss of generality we may assume that $f_{1}<f_{2}$. 
We begin with a general lemma about quadratic forms.

Definition 7.1. Let $Q$ be a form of discriminant $D<0$ and let $r \in \mathbb{Z}_{\geq 1}$. The form $Q^{\prime}$ is an $r$-lift of $Q$ if the following conditions hold:

(a) $Q$ and $Q^{\prime}$ have the same fundamental discriminant $d=d^{\prime}$.

(b) The discriminant of $Q^{\prime}$ satisfies $D^{\prime}=r^{2} D$.

(c) In the natural (restriction) map

$$
\phi: \mathrm{Cl}\left(D^{\prime}\right) \rightarrow \mathrm{Cl}(D)
$$

we have $\phi\left(\sigma^{\prime}\right)=\sigma$, where $\sigma \leftrightarrow Q$ and $\sigma^{\prime} \leftrightarrow Q^{\prime}$.

Lemma 7.2. Let $Q=\langle a, b, c\rangle$ be an $S L_{2}(\mathbb{Z})$-reduced form associated to $\sigma$. Then $\sigma$ has order dividing 2 if and only if $0=b$ or $b=a$ or $a=c$.

Suppose that $\sigma$ has order dividing 2 and $2 \mid D$. Then $Q$ has a 2-lift $Q^{\prime}$ with $Q^{\prime} \leftrightarrow \sigma^{\prime}$ of order 2 if and only if $0=b$.

Proof. Throughout this proof, we require only that forms be $S L_{2}(\mathbb{Z})$-reduced rather than $G L_{2}(\mathbb{Z})$-reduced, but we maintain all other assumptions on our forms, as in the introduction. Recall that $Q$ is $S L_{2}(\mathbb{Z})$-reduced if and only if $|b| \leq a \leq c$ and $b=0$ if either $|b|=a$ or $a=c$.

The first statement of the lemma is classical: The opposite of the form $Q$ is the form $S L_{2}(\mathbb{Z})$-equivalent to $Q^{\prime}=\langle a,-b, c\rangle$. But this form is already $S L_{2}(\mathbb{Z})$ reduced, unless $|b|=a$ or $a=c$, and in either of these cases in fact $Q^{\prime}$ is $S L_{2}(\mathbb{Z})$ equivalent to $Q$, so that $\sigma$ has order dividing 2 .

For the second statement, first suppose $0=b$ and that $a$ is odd. Note that the form $Q^{\prime}=\langle a, 0,4 c\rangle$ is a 2-lift of $Q$, since the set of primes which it represents is a subset of those represented by $Q$. If $c$ is odd, then a 2-lift is $\langle 4 a, 0, c\rangle$ if $4 a \leq c$ and $\langle c, 0,4 a\rangle$ if $4 a\rangle c$. This concludes this case, because if $a$ and $c$ are both even, then $Q$ is not primitive.

Next, suppose that $b=a$. Then since $D$ is even, $a$ is even, so $c$ is odd. Therefore a 2-lift of $Q$ is the form $S L_{2}(\mathbb{Z})$-equivalent to $Q^{\prime}=\langle 4 a, 2 a, c\rangle$, which is either $Q^{\prime}$ if $4 a<c$, or $\langle c,-2 a, 4 a\rangle$ if $2 a<c<4 a$, or $\langle c, 2(c-a), 4 a+c\rangle$ if $c<2 a$; we cannot have $4 a=c$ or $2 a=c$, as then $c$ is even and $Q$ is not primitive. In any case, the 2-lift visibly has order $>2$; therefore all 2-lifts have order $>2$ since they differ by an element of the kernel which is of order dividing 2, by Proposition A.1

Finally, suppose $a=c$. Here, we know that $b$ is even so $a$ is odd, and a 2-lift of $Q$ is the form $S L_{2}(\mathbb{Z})$-equivalent to $Q^{\prime}=\langle a, 2 b, 4 a\rangle$, which is $Q^{\prime}$ if $2 b<a$ and $\langle a, 2(b-a), 5 a-2 b\rangle$ if $2 b>a$; we cannot have $2 b=a$, since $a$ is odd. This form has order dividing 2 if and only if $b=a$ which is impossible ( $a$ must be even from the previous paragraph), and otherwise this lift has order $>2$.

Proposition 7.3. Let $Q_{1}, Q_{2}$ be forms with $d_{1}=d_{2}=d$ and $f_{1}<f_{2}$. Then $Q_{1} \sim Q_{2}$ if and only if $Q_{2}$ is the unique 2 - or the unique 4-lift of $Q_{1}$.

Proof. First, suppose that $f_{1} \mid f_{2}$ and that $A_{f_{1}}$ and $A_{f_{2}}$ have the same number of roots of unity. Note that the set of primes represented by $Q_{2}$ is contained in the set of primes represented by $Q_{1}$ up to a finite set if and only if $Q_{2}$ is an $r$-lift of $Q_{1}$ for some $r \in \mathbb{Z}_{>1}$. Moreover, if there exist two such $r$-lifts $Q_{2}, Q_{2}^{\prime}$, then these two forms will represent disjoint, infinite nonempty sets of primes. Putting these together, we see that $Q_{1} \sim Q_{2}$ if and only if $Q_{2}$ is the unique $r$-lift of $Q_{1}$ for some $r \in \mathbb{Z}_{>1}$. 
From Lemma 4.2 we have $\left[R_{2}: R_{1}\right] \in\{1,2\}$. On the other hand, by Lemma 4.3, $R_{1}$ and $R_{2}$ have the same genus class field, so from Corollary 2.10, if $p \nmid d$ is an odd prime, then $p \mid f_{1}$ if and only if $p \mid f_{2}$. From Lemma 2.13 we have

$$
\left[R_{2}: R_{1}\right]=\frac{h\left(D_{2}\right)}{h\left(D_{1}\right)}=u \frac{f_{2}}{f_{1}} \in\{1,2\}
$$

where

$$
u= \begin{cases}\left(1-\left(\frac{d}{2}\right) \frac{1}{2}\right), & \text { if } 2 \nmid f_{1} \text { and } 2 \mid f_{2} ; \\ 1, & \text { otherwise. }\end{cases}
$$

From Proposition 4.8, there exists a form $Q$ of discriminant $d f^{2}$ with $f=$ $\operatorname{gcd}\left(f_{1}, f_{2}\right)$ such that $Q_{1} \sim Q \sim Q_{2}$. But since $u \in \frac{1}{2} \mathbb{Z}$ we see from (*) that $f_{i} / f \in 2^{\mathbb{Z}}$ for $i=1,2$, so $f_{2} / f_{1} \in 2^{\mathbb{Z}}$ as well, and hence since $f_{1}<f_{2}$ we have $f=f_{1} \mid f_{2}$ and $Q=Q_{1}$. Moreover, we have $u=1 / 2$ or $u=1$, and hence either $f_{2}=2 f_{1}$ or $f_{2}=4 f_{1}$, so $Q_{2}$ is the unique 2 - or 4 -lift of $Q_{1}$.

To conclude, suppose that the two orders have different numbers of roots of unity. Then $d=-3,-4$ and $A_{f_{1}}$ is the maximal order and $A_{f_{2}}$ is not. Repeating the above analysis, we easily verify that either $f_{2}=2 f_{1}$ or $f_{2}=4 f_{1}$; the finitely many cases that can occur are listed in Table 6.

To conclude, from this proposition it suffices to give necessary and sufficient conditions for the form $Q_{1}$ to have a unique 2- or 4-lift. Note that if $Q_{2}$ is the unique 4-lift of $Q_{1}$, and $Q$ is the unique 2-lift of $Q_{1}$, then in fact $Q_{2}$ is the unique 2-lift of $Q$, and $Q_{1} \sim Q \sim Q_{2}$. Therefore it suffices to give criteria equivalent to those occurring in Proposition 4.8 .

Theorem 7.4. Let $Q_{1}=\left\langle a_{1}, b_{1}, c_{1}\right\rangle$ be a form. Then there exists a form $Q_{2} \sim Q_{1}$ such that $\left|D_{2}\right|>\left|D_{1}\right|$ and $d_{2}=d_{1}=d$ if and only if one of the following holds:

(i) $d \equiv 1(\bmod 8)$ and $2 \nmid D_{1}$;

(ii) $2 \mid D_{1}$ and either $b_{1}=a_{1}$ or $a_{1}=c_{1}$;

(iii) $d=-3$ and $Q_{1} \in\{\langle 1,1,1\rangle,\langle 1,0,3\rangle\}$;

(iv) $d=-4$ and $Q_{1}=\langle 1,0,1\rangle$.

Proof. If $d=-3$ or $d=-4$, we refer to Proposition 7.3 (and Table 6) and find cases (iii) and (iv).

More generally, we apply Proposition 4.8 The map $\phi$ is an isomorphism if and only if $h\left(D_{2}\right)=h\left(D_{1}\right)$. By Proposition A.1, this occurs if and only if $(d / 2)=1$ (and $f_{2}=2 f_{1}$ ), which is case (i).

For condition $(\dagger)$ from Proposition 4.8, first for any positive integer $f$, let

$$
C(f)=\frac{(A / f A)^{*}}{(\mathbb{Z} / f \mathbb{Z})^{*}}
$$

From the functoriality of the exact sequence of Lemma 2.13, we obtain a commutative diagram

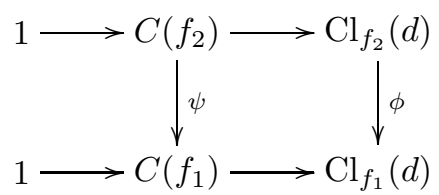


Now if $(\dagger)$ holds, then $C\left(f_{2}\right) \rightarrow C\left(f_{1}\right)$ is a nonsplit $\mathbb{Z} / 2 \mathbb{Z}$-extension, so we see from Proposition A.1 that $2 \mid D_{1}$. Therefore ( $\dagger$ ) holds if and only if $2 \mid D_{1}, \sigma_{1}$ has order 2 and $\sigma_{2}$ has order 4 . The result now follows from Lemma 7.2 .

\section{Computing Class groups}

To give an alternative proof of Proposition 5.1 , we may also characterize with at most one possible exception all imaginary quadratic extensions having class group of type dividing $(2, \ldots, 2,4)$. This result is not needed in the sequel, but it also yields an independent result (Theorem 8.2).

It was a classical problem to characterize field discriminants whose class group has exponent dividing 2, comprised of quadratic forms which are said to be "alone in their genus". It has long been known that the Brauer-Siegel theorem implies that there are only finitely many such discriminants $\mathrm{Ch}$.

Proposition 8.1 (Weinberger [We, Louboutin [Lo]). The number of discriminants $D=d f^{2}<0$ such that $\mathrm{Cl}_{f}(d)$ has exponent dividing 2 is finite. There are at least 65 and at most 66 such fundamental discriminants, and at least 36 and at most 37 such discriminants of nonmaximal orders.

Under the assumption of a suitable generalized Riemann hypothesis, there are exactly 65 and 36 of these discriminants, respectively.

The list of these discriminants can be found in [BS, Table 5]. Here we have a small variant of this problem, to which we may apply the same techniques.

Theorem 8.2. There are at least 226 and at most 227 fundamental discriminants $D=d$ such that $\mathrm{Cl}(d)$ is of type dividing $(2, \ldots, 2,4)$, and at least 199 and at most 205 such discriminants $D$ of nonmaximal orders.

These extensions are listed in Tables 7-16. Our proof of the proposition will again rely on the result of Tatuzawa (Lemma 2.14).

Lemma 8.3. There are effectively computable constants $C_{9}, C_{10}$, and $C_{11}$ satisfying the following condition:

With at most one exception, for all fundamental discriminants $d<0$ with $g$ distinct prime factors such that $|d| \geq C_{9}$ and $\mathrm{Cl}(d)$ is of type dividing $(2, \ldots, 2,4)$, we have $g \in\{10,11\}$ and $|d|<C_{g}$.

Proof. Let $d<0$ be a fundamental discriminant with $g$ distinct prime factors and class group of type dividing $(2, \ldots, 2,4)$. Recall as in the proof of Proposition 5.8 that $h(d) \leq 2^{g}$.

Let $C_{9}$ be the smallest positive integer such that

$$
2^{9}=512 \leq \frac{0.655}{\pi e} \frac{\sqrt{C_{9}}}{\log C_{9}}
$$

(allowable, since $\sqrt{x} / \log x$ is increasing for $x \geq e^{2}$ ). A calculation shows that $\log C_{9}>23$. Now apply Lemma 2.14 with $\epsilon=1 / \log C_{9}$. 
Suppose that $d$ is not the exceptional discriminant. Then if $|d| \geq C_{9}$, we have

$$
2^{g} \geq h(d)>\left(\frac{0.655}{\pi}\right) \frac{|d|^{1 / 2-1 / \log C_{9}}}{\log C_{9}} .
$$

In particular, this implies that

$$
2^{g}>\frac{0.655}{\pi e} \frac{\sqrt{C_{9}}}{\log C_{9}} \geq 2^{9}
$$

and therefore $g>9$.

By Lemma 5.7 we have $|d| \geq \widehat{d_{9}} \cdot 29^{g-9}$ and hence

$$
2^{g} \geq h(d)>\left(\frac{0.655}{\pi}\right) \frac{\widehat{d}_{9}^{1 / 2-1 / \log C_{9}}}{\log C_{9}}\left(29^{1 / 2-1 / \log C_{9}}\right)^{g-9} .
$$

This inequality implies that $g<12$.

For $t \in\{10,11\}$, let $C_{t}$ be the smallest positive integer such that

$$
2^{t} \leq\left(\frac{0.655}{\pi}\right) \frac{C_{t}^{1 / 2-1 / \log C_{9}}}{\log C_{9}} .
$$

Then if $|d| \geq C_{g}$,

$$
2^{g} \geq h(d)>\left(\frac{0.655}{\pi}\right) \frac{d^{1 / 2-1 / \log C_{9}}}{\log C_{9}} \geq\left(\frac{0.655}{\pi}\right) \frac{C_{g}^{1 / 2-1 / \log C_{9}}}{\log C_{9}} \geq 2^{g},
$$

a contradiction. This completes the proof.

We are now ready to prove the main result of this section.

Proof of Theorem 8.2. We have already computed (in the previous section) that there are exactly 226 such fundamental discriminants with $|d| \leq B$. Therefore the proposition will follow from Lemma 8.3 and Lemma 5.4 when it is shown that there are no fundamental discriminants $d<0$ with $\mathrm{Cl}(d)$ of type dividing $(2, \ldots, 2,4)$ satisfying one of the following conditions:

(1) $4 \cdot 67^{4}=B \leq|d|<C_{9}$; or

(2) the integer $d$ has exactly $g$ distinct prime divisors, $g \in\{10,11\}$ and $C_{9} \leq$ $|d|<C_{g}$.

Note that from the proof of Lemma 8.3 , we find $C_{9}=25593057435 \approx 2.5 \cdot 10^{10}$, $C_{10}=116145031943 \approx 1.1 \cdot 10^{11}$, and $C_{11}=527083115400 \approx 5.2 \cdot 10^{11}$.

The computations in (1) and (2) can be simplified by appealing to Corollary 5.3. if $p \leq \sqrt[4]{|d| / 4}$, then $(d / p) \neq 1$. We then test for each prime $p$ such that $\sqrt[4]{|d| / 4}<$ $p \leq \sqrt{|d| / 4}$ and $(d / p)=1$ if $\mathfrak{p}^{4}$ is principal (working in the group of quadratic forms of discriminant $d$ ), where $(p)=\mathfrak{p} \bar{p}$. To further rule out discriminants, we may also check, given two such primes $p_{1}, p_{2}$, that $\left(\mathfrak{p}_{1} \mathfrak{p}_{2}\right)^{2}$ is principal. For $d$ which satisfy all these conditions, we compute the class group $\mathrm{Cl}(d)$ itself (e.g. using an algorithm of Shanks) and check explicitly if it is of type dividing $(2, \ldots, 2,4)$. A computer search in MAGMA found no such $d$. (The code is available from the author by request.)

We also prove a complementary result which relies on a generalized Riemann hypothesis. 
Proposition 8.4. If the zeta function of the field $K=\mathbb{Q}(\sqrt{d})$ of discriminant $d<0$ does not have a zero in the interval $[1-(2 / \log |d|), 1)$ and the class group of $K$ is of type dividing $(2, \ldots, 2,4)$, then $|d|<1.3 \cdot 10^{10}$.

Proposition 8.5 (Louboutin $[\mathrm{LO})$. Let $K=\mathbb{Q}(\sqrt{d})$ be an imaginary quadratic field of discriminant d. Suppose that the zeta function of $K$ does not have a zero in the interval $[1-(2 / \log |d|), 1)$. Then

$$
h(d) \geq \frac{\pi}{3 e} \frac{\sqrt{|d|}}{\log |d|}
$$

where $e=\exp (1)$.

Proof of Proposition 8.4. We follow [Lo, Théorème 2]. Let $g$ be the number of distinct prime factors of the discriminant $d$. Then $\# \mathrm{Cl}(d)[2]=2^{g-1}$ so $h(d) \leq 2^{g}$. From Proposition 8.5. we see that $2^{g} \geq(\pi / 3 e) \sqrt{|d|} / \log |d|$. Recall that $|d| \geq \widehat{d_{t}}=$ $4 p_{1} \ldots p_{t-1}$ whenever $d \neq-3$. If we set

$$
t_{0}=\inf \left\{t \in \mathbb{Z}_{>0}: u \geq t \Rightarrow 2^{u}<\left(\frac{\pi}{3 e}\right) \frac{\left(\widehat{d}_{u}\right)^{1 / 2}}{\log \widehat{d}_{u}}\right\},
$$

then $|d|<\widehat{d}_{t_{0}}$ (see $\left[\mathrm{LO}\right.$ ). We compute easily that in this case $\widehat{d}_{t_{0}}=4 \cdot 3 \cdot \ldots \cdot 29<$ $1.3 \cdot 10^{10}$.

Theorem 8.6. Under the above Riemann hypothesis, there are exactly 226 fundamental discriminants $d$ such that $\mathrm{Cl}(d)$ is of type dividing $(2, \ldots, 2,4)$, and 199 such discriminants $D$ of nonmaximal orders.

This follows from Proposition 8.4 and the computations performed in the proof of Theorem 8.2

\section{APPENDIX: RING ClASS GROUPS}

In this appendix, we prove a proposition which characterizes ring class groups; we give a full statement for completeness.

Proposition A.1. For $f \in \mathbb{Z}_{>0}$, we have

$$
\frac{(A / f A)^{*}}{(\mathbb{Z} / f \mathbb{Z})^{*}} \cong \prod_{p^{e} \| f} \frac{\left(A / p^{e} A\right)^{*}}{\left(\mathbb{Z} / p^{e} \mathbb{Z}\right)^{*}}
$$

where $p$ is prime and $e>0$. We have

$$
\frac{\left(A / 2^{e} A\right)^{*}}{\left(\mathbb{Z} / 2^{e} \mathbb{Z}\right)^{*}} \cong \begin{cases}0, & \text { if } d \equiv 1(\bmod 8) \text { and } e=1, \\ \mathbb{Z} / 2 \mathbb{Z} \oplus \mathbb{Z} / 2^{e-2} \mathbb{Z}, & \text { if } d \equiv 1(\bmod 8) \text { and } e \geq 2, \\ \mathbb{Z} / 3 \mathbb{Z}, & \text { if } d \equiv 5(\bmod 8) \text { and } e=1, \\ \mathbb{Z} / 3 \mathbb{Z} \oplus \mathbb{Z} / 2 \mathbb{Z} \oplus \mathbb{Z} / 2^{e-2} \mathbb{Z}, & \text { if } d \equiv 5(\bmod 8) \text { and } e \geq 2, \\ \mathbb{Z} / 2 \mathbb{Z} \oplus \mathbb{Z} / 2^{e-1} \mathbb{Z}, & \text { if } d \equiv 4(\bmod 8), \\ \mathbb{Z} / 2^{e} \mathbb{Z}, & \text { if } d \equiv 0(\bmod 8),\end{cases}
$$

and

$$
\frac{\left(A / 3^{e} A\right)^{*}}{\left(\mathbb{Z} / 3^{e} \mathbb{Z}\right)^{*}} \cong \begin{cases}\mathbb{Z} / 2 \mathbb{Z} \oplus \mathbb{Z} / 3^{e-1} \mathbb{Z}, & \text { if } d \equiv 1(\bmod 3) \\ \mathbb{Z} / 4 \mathbb{Z} \oplus \mathbb{Z} / 3^{e-1} \mathbb{Z}, & \text { if } d \equiv 2(\bmod 3) \\ \mathbb{Z} / 3^{e} \mathbb{Z}, & \text { if } d \equiv 3(\bmod 9) \\ \mathbb{Z} / 3 \mathbb{Z} \oplus \mathbb{Z} / 3^{e-1} \mathbb{Z}, & \text { if } d \equiv 6(\bmod 9)\end{cases}
$$


and finally for $p \neq 2,3$, we have

$$
\frac{\left(A / p^{e} A\right)^{*}}{\left(\mathbb{Z} / p^{e} \mathbb{Z}\right)^{*}} \cong \begin{cases}\mathbb{Z} /(p-1) \mathbb{Z} \oplus \mathbb{Z} / p^{e-1} \mathbb{Z}, & \text { if }(d / p)=1, \\ \mathbb{Z} /(p+1) \mathbb{Z} \oplus \mathbb{Z} / p^{e-1} \mathbb{Z}, & \text { if }(d / p)=-1, \\ \mathbb{Z} / p^{e} \mathbb{Z}, & \text { if }(d / p)=0 .\end{cases}
$$

Proof. The first statement follows from the Chinese remainder theorem. From Lemma 2.13, we have

$$
\# \frac{\left(A / p^{e} A\right)^{*}}{\left(\mathbb{Z} / p^{e} \mathbb{Z}\right)^{*}}=p^{e}\left(1-\left(\frac{d}{p}\right) \frac{1}{p}\right) .
$$

We first treat the trivial case $p^{e}=2$ : then $(\mathbb{Z} / 2 \mathbb{Z})^{*}$ is the trivial group, and

$$
(A / 2 A)^{*} \cong \begin{cases}\mathbb{Z} / 3 \mathbb{Z}, & \text { if }(d / 2)=-1 \\ \mathbb{Z} / 2 \mathbb{Z}, & \text { if }(d / 2)=0 \\ 0, & \text { if }(d / 2)=1\end{cases}
$$

Note that

$$
\frac{\left(A / p^{e} A\right)^{*}}{\left(\mathbb{Z} / p^{e} \mathbb{Z}\right)^{*}} \cong \frac{\left(A_{p} / p^{e} A_{p}\right)^{*}}{\left(\mathbb{Z}_{p} / p^{e} \mathbb{Z}_{p}\right)^{*}},
$$

where $A_{p}$ denotes the completion of $A$ at $p$ and $\mathbb{Z}_{p}$ the ring of $p$-adic integers. So if $(d / p)=1$, by [N, $\S I I .5]$ we have

$$
\frac{\left(A_{p} / p^{e} A_{p}\right)^{*}}{\left(\mathbb{Z}_{p} / p^{e} \mathbb{Z}_{p}\right)^{*}} \cong\left(\mathbb{Z}_{p} / p^{e} \mathbb{Z}_{p}\right)^{*}= \begin{cases}0, & \text { if } p^{e}=2, \\ \mathbb{Z} / 2 \mathbb{Z} \oplus \mathbb{Z} / 2^{e-2} \mathbb{Z}, & \text { if } p=2 \text { and } e \geq 2, \\ \mathbb{Z} /(p-1) \mathbb{Z} \oplus \mathbb{Z} / p^{e-1} \mathbb{Z}, & \text { otherwise. }\end{cases}
$$

From now on we assume $p^{e} \neq 2$ and $(d / p) \neq 1$.

Let $K_{p}$ denote the completion of $K$ at $p$, so that $A_{p}$ is its valuation ring with maximal ideal $\mathfrak{p}$ and uniformizer $\pi$. We denote by $v$ the unique valuation on $K_{p}$ normalized so that $v(p)=1$. Let

$$
V\left(A_{p}\right)=\left\{x \in A_{p}: v(x)>1 /(p-1)\right\} .
$$

It follows from [N] Proposition II.5.4] that there exists a (continuous) homomorphism $\log _{p}: A_{p}^{*} \rightarrow A_{p}$, with the property that $\log _{p}$ restricts to an isomorphism $1+V\left(A_{p}\right) \stackrel{\sim}{\longrightarrow} V\left(A_{p}\right)$.

One has an exact sequence

$$
0 \rightarrow \frac{1+V\left(A_{p}\right)}{1+p^{e} A_{p}} \rightarrow\left(\frac{A_{p}}{p^{e} A_{p}}\right)^{*} \rightarrow \frac{A_{p}^{*}}{1+V\left(A_{p}\right)} \rightarrow 0 .
$$

We have an analogous exact sequence for $\mathbb{Z}_{p}$, and since $\left(1+V\left(A_{p}\right)\right) \cap \mathbb{Z}_{p}=1+V\left(\mathbb{Z}_{p}\right)$, it injects term-by-term into the one for $A_{p}$, yielding the following exact sequence:

$$
0 \rightarrow \frac{\frac{1+V\left(A_{p}\right)}{1+p^{e} A_{p}}}{\frac{1+V\left(\mathbb{Z}_{p}\right)}{1+p^{e} \mathbb{Z}_{p}}} \rightarrow \frac{\left(\frac{A_{p}}{p^{e} A_{p}}\right)^{*}}{\left(\frac{\mathbb{Z}_{p}}{p^{e} \mathbb{Z}_{p}}\right)^{*}} \stackrel{\phi}{\rightarrow} \frac{\frac{A_{p}^{*}}{1+V\left(A_{p}\right)}}{\frac{\mathbb{Z}_{p}^{*}}{1+V\left(\mathbb{Z}_{p}\right)}} \rightarrow 0 .
$$

From the above, we see that by the logarithm map,

$$
\frac{1+V\left(A_{p}\right)}{1+p^{e} A_{p}} \cong \frac{V\left(A_{p}\right)}{p^{e} A_{p}} \quad \text { and } \quad \frac{1+V\left(\mathbb{Z}_{p}\right)}{1+p^{e} \mathbb{Z}_{p}} \cong \frac{V\left(\mathbb{Z}_{p}\right)}{p^{e} \mathbb{Z}_{p}}
$$


Now let us assume that $p \neq 2,3$. Then $V\left(A_{p}\right)=\mathfrak{p}$, and $V\left(\mathbb{Z}_{p}\right)=p \mathbb{Z}_{p}$. We first analyze the group

$$
\operatorname{ker} \phi=\frac{V\left(A_{p}\right) / p^{e} A_{p}}{V\left(\mathbb{Z}_{p}\right) / p^{e} \mathbb{Z}_{p}}
$$

from $(\diamond)$; we claim it is cyclic. If $(d / p)=-1$, with $\epsilon \in A_{p}$ such that $A_{p}=\mathbb{Z}_{p}+\epsilon \mathbb{Z}_{p}$ as additive groups, then since $\mathfrak{p}=V\left(A_{p}\right)=p \mathbb{Z}_{p}+p \epsilon \mathbb{Z}_{p}$, the element $p \epsilon$ generates the group $\operatorname{ker} \phi$. If $(d / p)=0$, then $V\left(A_{p}\right)=\pi \mathbb{Z}_{p}+p \mathbb{Z}_{p}$, so $\pi$ is a generator. It follows that

$$
\operatorname{ker} \phi \cong \begin{cases}\mathbb{Z} / p^{e-1} \mathbb{Z}, & \text { if }(d / p)=-1 \\ \mathbb{Z} / p^{e} \mathbb{Z}, & \text { if }(d / p)=0\end{cases}
$$

Now we analyze the image of $\phi$. We have $A_{p}^{*} /\left(1+V\left(A_{p}\right)\right) \cong \mu\left(A_{p}\right)$ (see [N] Proposition II.5.3]), and this group can be computed as follows. Since $\left[K_{p}: \mathbb{Q}_{p}\right]=2$ and the extension $\mathbb{Q}_{p}\left(\zeta_{p}\right)$ is a totally ramified extension of $\mathbb{Q}_{p}$ of degree $p-1$, we conclude that $A_{p}$ contains no $p$-power roots of unity. Therefore

$$
\mu\left(A_{p}\right) \cong \begin{cases}\mathbb{Z} /\left(p^{2}-1\right) \mathbb{Z}, & \text { if }(d / p)=-1, \\ \mathbb{Z} /(p-1) \mathbb{Z}, & \text { if }(d / p)=0\end{cases}
$$

Since $\mu\left(\mathbb{Z}_{p}\right) \cong \mathbb{Z} /(p-1) \mathbb{Z}$, putting these two pieces together, we see that in the exact sequence $(\diamond)$, the kernel and image groups have orders which are relatively prime to each other and hence the exact sequence splits, and we obtain the result of the proposition.

To conclude, we must treat the cases $p=2, p=3$. Every field extension of $\mathbb{Q}_{2}$ of degree 2 is isomorphic to $\mathbb{Q}_{2}(\sqrt{c})$ for $c \in\{-1, \pm 2, \pm 3, \pm 6\}$, and similarly for $\mathbb{Q}_{3}$ we have $c \in\{-1,-3,3$,$\} . We leave it to the reader to verify the following: For p=2$,

$$
\frac{\left(A / 2^{e} A\right)^{*}}{\left(\mathbb{Z} / 2^{e} \mathbb{Z}\right)^{*}} \cong \begin{cases}\langle(-1+\sqrt{-3}) / 2\rangle \times\langle\sqrt{-3}\rangle \times\langle 1+2 \sqrt{-3}\rangle, & \text { if } c=-3, \\ \langle\sqrt{-1}\rangle \times\langle 1+2 \sqrt{-1}\rangle, & \text { if } c=-1, \\ \langle 1+2 \sqrt{c}\rangle, & \text { if } c=3, \\ \langle 1+\sqrt{c}\rangle, & \text { if } 2 \mid c,\end{cases}
$$

and for $p=3$,

$$
\frac{\left(A / 3^{e} A\right)^{*}}{\left(\mathbb{Z} / 3^{e} \mathbb{Z}\right)^{*}} \cong \begin{cases}\langle 1+\sqrt{-1}\rangle \times\langle 1+3 \sqrt{-1}\rangle, & \text { if } c=-1, \\ \langle 1+\sqrt{3}\rangle, & \text { if } c=3, \\ \langle(1+\sqrt{-3}) / 2\rangle \times\langle 1+3 \sqrt{-3}\rangle, & \text { if } c=-3 .\end{cases}
$$

Computing the orders of these elements yields the conclusion of the proposition.

\section{TABLES}

In Tables 1 and 2, we list equivalence classes with two fundamental discriminants $(\delta(C)=2)$, then in Tables 3-5 those with three fundamental discriminants, then in Table 6 the exceptional cases with one fundamental discriminant (see Theorem 7.4(iii)-(iv)). Within each table, the classes are sorted by the smallest fundamental discriminant $d$ in each class. Every form in an equivalence class has associated to it the same genus class field $P$ (Lemma 4.3), denoted $\mathbb{Q}\left[a_{1}, \ldots, a_{r}\right]=$ $\mathbb{Q}\left(\sqrt{a_{1}}, \ldots, \sqrt{a_{r}}\right)$. The class group $\mathrm{Cl}_{f}(d)$ for each form is given by its type. The set $E$ denotes the exceptional set for each equivalence class (6.3). 
If $r \in \mathbb{Z}_{\geq 0}$, an abelian group $G$ is said to be of type $(\underbrace{2, \ldots, 2}_{r}, 4)$ if

$$
G \cong(\mathbb{Z} / 2 \mathbb{Z})^{r} \oplus \mathbb{Z} / 4 \mathbb{Z}
$$

The group $G$ is of type dividing $(2, \ldots, 2,4)$ if there is an injection of groups

$$
G \hookrightarrow(\mathbb{Z} / 2 \mathbb{Z})^{r} \oplus \mathbb{Z} / 4 \mathbb{Z}
$$

for some $r \in \mathbb{Z}_{\geq 0}$.

In Tables $7-16$, we list the orders of imaginary quadratic fields with class group of type dividing $(2, \ldots, 2,4)$, with at most one possible exception (as in Theorem 8.2). In particular, there is no order with class group of type $(2,2,2,2,2)$ (unless this is the one exception!). The tables are sorted by the isomorphism class of the class group, and within each table the classes are first sorted by fundamental discriminant and then discriminant.

\begin{tabular}{|c|c|c|c|c|c|c|}
\hline$Q$ & $|D|$ & $|d|$ & $f$ & $P$ & $\mathrm{Cl}_{f}(d)$ & $E$ \\
\hline$\overline{\langle 1,0,5\rangle}$ & 20 & 20 & 1 & $\mathbb{Q}[-1,5]$ & $(2)$ & $\{5\}$ \\
\hline$\langle 1,0,25\rangle$ & 100 & 4 & 5 & & (2) & $\emptyset$ \\
\hline$\langle 1,0,8\rangle$ & 32 & 8 & 2 & $\mathbb{Q}[-1,2]$ & $(2)$ & $\emptyset$ \\
\hline$\langle 1,0,16\rangle$ & 64 & 4 & 4 & & (2) & $\emptyset$ \\
\hline$\langle 1,0,9\rangle$ & 36 & 4 & 3 & $\mathbb{Q}[-1,-3]$ & $(2)$ & $\emptyset$ \\
\hline$\langle 1,0,12\rangle$ & 48 & 3 & 4 & & $(2)$ & $\emptyset$ \\
\hline$\langle 5,0,6\rangle$ & 120 & 120 & 1 & $\mathbb{Q}[2,-3,5]$ & $(2,2)$ & $\{5\}$ \\
\hline$\langle 11,4,14\rangle$ & 600 & 24 & 5 & & $(2,4)$ & $\emptyset$ \\
\hline$\langle 5,0,8\rangle$ & 160 & 40 & 2 & $\mathbb{Q}[-1,2,5]$ & $(2,2)$ & $\{5\}$ \\
\hline$\langle 13,8,32\rangle$ & 1600 & 4 & 20 & & $(2,4)$ & $\emptyset$ \\
\hline$\langle 1,0,45\rangle$ & 180 & 20 & 3 & $\mathbb{Q}[-1,-3,5]$ & $(2,2)$ & $\emptyset$ \\
\hline$\langle 1,0,60\rangle$ & 240 & 15 & 4 & & $(2,2)$ & $\emptyset$ \\
\hline$\langle 5,0,9\rangle$ & 180 & 20 & 3 & $\mathbb{Q}[-1,-3,5]$ & $(2,2)$ & $\{5\}$ \\
\hline$\langle 9,6,26\rangle$ & 900 & 4 & 15 & & $(2,4)$ & $\emptyset$ \\
\hline$\langle 8,0,9\rangle$ & 288 & 8 & 6 & $\mathbb{Q}[-1,2,-3]$ & $(2,2)$ & $\emptyset$ \\
\hline$\langle 9,6,17\rangle$ & 576 & 4 & 12 & & $(2,4)$ & $\emptyset$ \\
\hline$\langle 1,0,120\rangle$ & 480 & 120 & 2 & $\mathbb{Q}[-1,2,-3,5]$ & $(2,2,2)$ & $\emptyset$ \\
\hline$\langle 1,0,240\rangle$ & 960 & 15 & 8 & & $(2,2,2)$ & $\emptyset$ \\
\hline$\langle 5,0,24\rangle$ & 480 & 120 & 2 & $\mathbb{Q}[-1,2,-3,5]$ & $(2,2,2)$ & $\{5\}$ \\
\hline$\langle 21,6,29\rangle$ & 2400 & 24 & 10 & & $(2,2,4)$ & $\emptyset$ \\
\hline$\langle 3,0,40\rangle$ & 480 & 120 & 2 & $\mathbb{Q}[-1,2,-3,5]$ & $(2,2,2)$ & $\{3\}$ \\
\hline$\langle 27,12,28\rangle$ & 2880 & 20 & 12 & & $(2,2,4)$ & $\emptyset$ \\
\hline$\langle 3,0,56\rangle$ & 672 & 168 & 2 & $\mathbb{Q}[-1,2,-3,-7]$ & $(2,2,2)$ & $\{3\}$ \\
\hline$\langle 20,12,27\rangle$ & 2016 & 56 & 6 & & $(2,2,4)$ & $\emptyset$ \\
\hline$\langle 8,0,21\rangle$ & 672 & 168 & 2 & $\mathbb{Q}[-1,2,-3,-7]$ & $(2,2,2)$ & $\emptyset$ \\
\hline$\langle 29,12,36\rangle$ & 4032 & 7 & 24 & & $(2,2,4)$ & $\emptyset$ \\
\hline$\langle 3,0,80\rangle$ & 960 & 15 & 8 & $\mathbb{Q}[-1,2,-3,5]$ & $(2,2,2)$ & $\{3\}$ \\
\hline$\langle 27,24,32\rangle$ & 2880 & 20 & 12 & & $(2,2,4)$ & $\emptyset$ \\
\hline$\langle 7,6,39\rangle$ & 1056 & 264 & 2 & $\mathbb{Q}[-1,2,-3,-11]$ & $(2,2,4)$ & $\emptyset$ \\
\hline$\langle 7,4,76\rangle$ & 2112 & 132 & 4 & & $(2,2,4)$ & $\emptyset$ \\
\hline$\langle 15,12,20\rangle$ & 1056 & 264 & 2 & $\mathbb{Q}[-1,2,-3,-11]$ & $(2,2,4)$ & $\emptyset$ \\
\hline$\langle 23,12,36\rangle$ & 3168 & 88 & 6 & & $(2,2,4)$ & $\emptyset$ \\
\hline
\end{tabular}

TABle 1. Equivalence classes $C$ of forms $(\# \delta(C)=2, \# C=2), 1$ of 2 
TABle 1. (continued)

\begin{tabular}{|c|c|c|c|c|c|c|}
\hline$Q$ & $|D|$ & $|d|$ & $f$ & $P$ & $\mathrm{Cl}_{f}(d)$ & $E$ \\
\hline 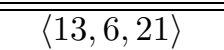 & $\overline{1056}$ & $\overline{264}$ & $\overline{2}$ & $\overline{\mathbb{Q}[-1,2,-3,-11]}$ & $\overline{(2,2,4)}$ & $\bar{\theta}$ \\
\hline$\langle 13,2,61\rangle$ & 3168 & 88 & 6 & & $(2,2,4)$ & $\emptyset$ \\
\hline$\langle 8,0,39\rangle$ & 1248 & 312 & 2 & $\mathbb{Q}[-1,2,-3,13]$ & $(2,2,2)$ & $\emptyset$ \\
\hline$\langle 15,12,44\rangle$ & 2496 & 39 & 8 & & $(2,2,4)$ & $\emptyset$ \\
\hline$\langle 5,4,68\rangle$ & 1344 & 84 & 4 & $\mathbb{Q}[-1,2,-3,-7]$ & $(2,2,4)$ & $\emptyset$ \\
\hline$\langle 5,2,101\rangle$ & 2016 & 56 & 6 & & $(2,2,4)$ & $\emptyset$ \\
\hline$\langle 11,8,32\rangle$ & 1344 & 84 & 4 & $\mathbb{Q}[-1,2,-3,-7]$ & $(2,2,4)$ & $\emptyset$ \\
\hline$\langle 11,4,92\rangle$ & 4032 & 7 & 24 & & $(2,2,4)$ & $\emptyset$ \\
\hline$\langle 20,4,23\rangle$ & 1824 & 456 & 2 & $\overline{\mathbb{Q}}[-1,2,-3,-19]$ & $(2,2,4)$ & $\emptyset$ \\
\hline$\langle 23,20,44\rangle$ & 3648 & 228 & 4 & & $(2,2,4)$ & $\emptyset$ \\
\hline$\langle 19,4,28\rangle$ & 2112 & 132 & 4 & $\mathbb{Q}[-1,2,-3,-11]$ & $(2,2,4)$ & $\emptyset$ \\
\hline$\langle 19,10,43\rangle$ & 3168 & 88 & 6 & & $(2,2,4)$ & $\emptyset$ \\
\hline$\langle 8,0,105\rangle$ & 3360 & 840 & 2 & $\mathbb{Q}[-1,2,-3,5,-7]$ & $(2,2,2,2)$ & $\phi$ \\
\hline$\langle 32,24,57\rangle$ & 6720 & 420 & 4 & & $(2,2,2,4)$ & $\emptyset$ \\
\hline$\langle 21,0,40\rangle$ & 3360 & 840 & 2 & $\mathbb{Q}[-1,2,-3,5,-7]$ & $(2,2,2,2)$ & $\emptyset$ \\
\hline$\langle 45,30,61\rangle$ & 10080 & 280 & 6 & & $(2,2,2,4)$ & $\emptyset$ \\
\hline$\langle 24,0,55\rangle$ & 5280 & 1320 & 2 & $\mathbb{Q}[-1,2,-3,5,-11]$ & $(2,2,2,2)$ & $\phi$ \\
\hline$\langle 39,36,76\rangle$ & 10560 & 660 & 4 & & $(2,2,2,4)$ & $\emptyset$ \\
\hline$\langle 33,0,40\rangle$ & 5280 & 1320 & 2 & $\mathbb{Q}[-1,2,-3$, & $(2,2,2,2)$ & $\emptyset$ \\
\hline$\langle 52,36,57\rangle$ & 10560 & 660 & 4 & & $(2,2,2,4)$ & $\emptyset$ \\
\hline$\langle 23,4,68\rangle$ & 6240 & 1560 & 2 & $\mathbb{Q}[-1,2,-3,5,13]$ & $(2,2,2,4)$ & $\emptyset$ \\
\hline$\langle 23,18,207\rangle$ & 18720 & 520 & 6 & & $(2,2,2,4)$ & $\emptyset$ \\
\hline$\langle 28,12,57\rangle$ & 6240 & 1560 & 2 & $\mathbb{Q}[-1,2,-3,5,13]$ & $(2,2,2,4)$ & $\emptyset$ \\
\hline$\langle 72,48,73\rangle$ & 18720 & 520 & 6 & & $(2,2,2,4)$ & $\emptyset$ \\
\hline$\langle 21,12,76\rangle$ & 6240 & 1560 & 2 & $\mathbb{Q}[-1,2,-3,5,13]$ & $(2,2,2,4)$ & $\emptyset$ \\
\hline$\langle 45,30,109\rangle$ & 18720 & 520 & 6 & & $(2,2,2,4)$ & $\emptyset$ \\
\hline$\langle 35,30,51\rangle$ & 6240 & 1560 & 2 & $\mathbb{Q}[-1,2,-3,5,13]$ & $(2,2,2,4)$ & $\emptyset$ \\
\hline$\langle 36,12,131\rangle$ & 18720 & 520 & 6 & & $(2,2,2,4)$ & $\emptyset$ \\
\hline$\langle 19,14,91\rangle$ & 6720 & 420 & 4 & $\mathbb{Q}[-1,2,-3,5,-7]$ & $(2,2,2,4)$ & $\phi$ \\
\hline$\langle 19,16,136\rangle$ & 10080 & 280 & 6 & & $(2,2,2,4)$ & $\emptyset$ \\
\hline$\langle 28,20,85\rangle$ & 9120 & 2280 & 2 & $\mathbb{Q}[-1,2,-3,5,-19]$ & $(2,2,2,4)$ & $\emptyset$ \\
\hline$\langle 45,30,157\rangle$ & 27360 & 760 & 6 & & $(2,2,2,4)$ & $\emptyset$ \\
\hline$\langle 51,48,56\rangle$ & 9120 & 2280 & 2 & $\mathbb{Q}[-1,2,-3,5,-19]$ & $(2,2,2,4)$ & $\emptyset$ \\
\hline$\langle 59,4,116\rangle$ & 27360 & 760 & 6 & & $(2,2,2,4)$ & $\emptyset$ \\
\hline$\langle 33,24,88\rangle$ & 11040 & 2760 & 2 & $\mathbb{Q}[-1,2,-3,5,-23]$ & $(2,2,2,4)$ & $\emptyset$ \\
\hline$\langle 57,6,97\rangle$ & 22080 & 1380 & 4 & & $(2,2,2,4)$ & $\emptyset$ \\
\hline$\langle 39,6,71\rangle$ & 11040 & 2760 & 2 & $\mathbb{Q}[-1,2,-3,5,-23]$ & $(2,2,2,4)$ & $\emptyset$ \\
\hline$\langle 71,70,95\rangle$ & 22080 & 1380 & 4 & & $(2,2,2,4)$ & $\emptyset$ \\
\hline$\langle 76,20,145\rangle$ & 43680 & 10920 & 2 & $\mathbb{Q}[-1,2,-3,5,-7,13]$ & $(2,2,2,2,4)$ & $\emptyset$ \\
\hline$\langle 96,72,241\rangle$ & 87360 & 5460 & 4 & & $(2,2,2,2,4)$ & $\emptyset$ \\
\hline$\langle 88,32,127\rangle$ & 43680 & 10920 & 2 & $\mathbb{Q}[-1,2,-3,5,-7,13]$ & $(2,2,2,2,4)$ & $\emptyset$ \\
\hline$\langle 127,4,172\rangle$ & 87360 & 5460 & 4 & & $(2,2,2,2,4)$ & $\emptyset$ \\
\hline$\langle 57,18,193\rangle$ & 43680 & 10920 & 2 & $\mathbb{Q}[-1,2,-3,5,-7,13]$ & $(2,2,2,2,4)$ & $\emptyset$ \\
\hline$\langle 148,132,177\rangle$ & 87360 & 5460 & 4 & & $(2,2,2,2,4)$ & $\emptyset$ \\
\hline$\langle 55,10,199\rangle$ & 43680 & 10920 & 2 & $\mathbb{Q}[-1,2,-3,5,-7,13]$ & $(2,2,2,2,4)$ & $\bar{\emptyset}$ \\
\hline$\langle 159,120,160\rangle$ & 87360 & 5460 & 4 & & $(2,2,2,2,4)$ & $\emptyset$ \\
\hline
\end{tabular}


TABle 2. Equivalence classes $C$ of forms $(\# \delta(C)=2, \# C=3), 1$ of 2

\begin{tabular}{|c|c|c|c|c|c|c|}
\hline$Q$ & $|D|$ & $|d|$ & $f$ & $P$ & $\mathrm{Cl}_{f}(d)$ & $E$ \\
\hline$\overline{\langle\langle 1,1,4\rangle}$ & $\overline{15}$ & 15 & $\overline{1} 1$ & $\overline{\mathbb{Q}}[-3,5]$ & 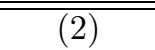 & $\overline{\phi \emptyset}$ \\
\hline$\langle 1,0,15\rangle$ & 60 & 15 & 2 & & (2) & $\emptyset$ \\
\hline$\langle 1,1,19\rangle$ & 75 & 3 & 5 & & (2) & $\emptyset$ \\
\hline$\langle 2,2,11\rangle$ & 84 & 84 & 1 & $\mathbb{Q}[-1,-3,-7]$ & $(2,2)$ & $\overline{\{2\}}$ \\
\hline$\langle 8,4,11\rangle$ & 336 & 84 & 2 & & $(2,4)$ & $\emptyset$ \\
\hline$\langle 11,2,23\rangle$ & 1008 & 7 & 12 & & $(2,4)$ & $\emptyset$ \\
\hline$\langle 3,0,8\rangle$ & 96 & 24 & 2 & $\mathbb{Q}[-1,2,-3]$ & $(2,2)$ & $\{3\}$ \\
\hline$\langle 8,8,11\rangle$ & 288 & 8 & 6 & & $(2,2)$ & $\emptyset$ \\
\hline$\langle 11,6,27\rangle$ & 1152 & 8 & 12 & & $(2,4)$ & $\emptyset$ \\
\hline$\langle 5,2,5\rangle$ & 96 & 24 & 2 & $\mathbb{Q}[-1,2,-3]$ & $(2,2)$ & $\emptyset$ \\
\hline$\langle 5,4,20\rangle$ & 384 & 24 & 4 & & $(2,4)$ & $\emptyset$ \\
\hline$\langle 5,2,29\rangle$ & 576 & 4 & 12 & & $(2,4)$ & $\emptyset$ \\
\hline$\langle 7,6,7\rangle$ & 160 & 40 & 2 & $\mathbb{Q}[-1,2,5]$ & $(2,2)$ & $\emptyset$ \\
\hline$\langle 7,2,23\rangle$ & 640 & 40 & 4 & & $(2,4)$ & $\emptyset$ \\
\hline$\langle 7,4,12\rangle$ & 320 & 20 & 4 & & $(2,4)$ & $\emptyset$ \\
\hline$\langle 2,2,23\rangle$ & 180 & 20 & 3 & $\mathbb{Q}[-1,-3,5]$ & $(2,2)$ & $\{2\}$ \\
\hline$\langle 8,4,23\rangle$ & 720 & 20 & 6 & & $(2,4)$ & $\emptyset$ \\
\hline$\langle 3,0,20\rangle$ & 240 & 15 & 4 & & $(2,2)$ & $\{3\}$ \\
\hline$\langle 3,0,16\rangle$ & 192 & 3 & 8 & $\mathbb{Q}[-1,2,-3]$ & $(2,2)$ & $\{3\}$ \\
\hline$\langle 4,4,19\rangle$ & 288 & 8 & 6 & & $(2,2)$ & $\emptyset$ \\
\hline$\langle 16,8,19\rangle$ & 1152 & 8 & 12 & & $(2,4)$ & $\emptyset$ \\
\hline$\langle 6,6,19\rangle$ & 420 & 420 & 1 & $\mathbb{Q}[-1,-3,5,-7]$ & $(2,2,2)$ & $\emptyset$ \\
\hline$\langle 19,12,24\rangle$ & 1680 & 420 & 2 & & $(2,2,4)$ & $\emptyset$ \\
\hline$\langle 19,16,31\rangle$ & 2100 & 84 & 5 & & $(2,2,4)$ & $\emptyset$ \\
\hline$\langle 11,8,11\rangle$ & 420 & 420 & 1 & $\mathbb{Q}[-1,-3,5,-7]$ & $(2,2,2)$ & $\emptyset$ \\
\hline$\langle 11,6,39\rangle$ & 1680 & 420 & 2 & & $(2,2,4)$ & $\emptyset$ \\
\hline$\langle 11,10,50\rangle$ & 2100 & 84 & 5 & & $(2,2,4)$ & $\emptyset$ \\
\hline$\langle 4,4,31\rangle$ & 480 & 120 & 2 & $\mathbb{Q}[-1,2,-3,5]$ & $(2,2,2)$ & $\emptyset$ \\
\hline$\langle 16,8,31\rangle$ & 1920 & 120 & 4 & & $(2,2,4)$ & $\emptyset$ \\
\hline$\langle 15,0,16\rangle$ & 960 & 15 & 8 & & $(2,2,2)$ & $\emptyset$ \\
\hline$\langle 12,12,13\rangle$ & 480 & 120 & 2 & $\mathbb{Q}[-1,2,-3,5]$ & $(2,2,2)$ & $\emptyset$ \\
\hline$\langle 13,2,37\rangle$ & 1920 & 120 & 4 & & $(2,2,4)$ & $\emptyset$ \\
\hline$\langle 13,4,28\rangle$ & 1440 & 40 & 6 & & $(2,2,4)$ & $\emptyset$ \\
\hline$\langle 12,12,17\rangle$ & 672 & 168 & 2 & $\mathbb{Q}[-1,2,-3,-7]$ & $(2,2,2)$ & $\emptyset$ \\
\hline$\langle 17,10,41\rangle$ & 2688 & 168 & 4 & & $(2,2,4)$ & $\emptyset$ \\
\hline$\langle 17,4,20\rangle$ & 1344 & 84 & 4 & & $(2,2,4)$ & $\emptyset$ \\
\hline$\langle 13,2,13\rangle$ & 672 & 168 & 2 & $\mathbb{Q}[-1,2,-3,-7]$ & $(2,2,2)$ & $\phi$ \\
\hline$\langle 13,4,52\rangle$ & 2688 & 168 & 4 & & $(2,2,4)$ & $\emptyset$ \\
\hline$\langle 13,8,40\rangle$ & 2016 & 56 & 6 & & $(2,2,4)$ & $\emptyset$ \\
\hline$\langle 8,8,41\rangle$ & 1248 & 312 & 2 & $\mathbb{Q}[-1,2,-3,13]$ & $(2,2,2)$ & $\emptyset$ \\
\hline$\langle 32,16,41\rangle$ & 4992 & 312 & 4 & & $(2,2,4)$ & $\emptyset$ \\
\hline$\langle 20,12,33\rangle$ & 2496 & 39 & 8 & & $(2,2,4)$ & $\emptyset$ \\
\hline$\langle 12,12,73\rangle$ & 3360 & 840 & 2 & $\mathbb{Q}[-1,2,-3,5,-7]$ & $(2,2,2,2)$ & $\emptyset$ \\
\hline$\langle 48,24,73\rangle$ & 13440 & 840 & 4 & & $(2,2,2,4)$ & $\emptyset$ \\
\hline$\langle 33,12,52\rangle$ & 6720 & 420 & 4 & & $(2,2,2,4)$ & $\emptyset$ \\
\hline
\end{tabular}


TABLE 2. (continued)

\begin{tabular}{cccc|cc|c}
$Q$ & $|D|$ & $|d|$ & $f$ & $P$ & $\mathrm{Cl}_{f}(d)$ & $E$ \\
\hline \hline$\langle 31,22,31\rangle$ & 3360 & 840 & 2 & $\mathbb{Q}[-1,2,-3,5,-7]$ & $(2,2,2,2)$ & $\emptyset$ \\
$\langle 31,18,111\rangle$ & 13440 & 840 & 4 & & $(2,2,2,4)$ & $\emptyset$ \\
$\langle 31,10,55\rangle$ & 6720 & 420 & 4 & & $(2,2,2,4)$ & $\emptyset$ \\
\hline$\langle 20,20,47\rangle$ & 3360 & 840 & 2 & $\mathbb{Q}[-1,2,-3,5,-7]$ & $(2,2,2,2)$ & $\emptyset$ \\
$\langle 47,40,80\rangle$ & 13440 & 840 & 4 & & $(2,2,2,4)$ & $\emptyset$ \\
$\langle 47,42,63\rangle$ & 10080 & 280 & 6 & & $(2,2,2,4)$ & $\emptyset$ \\
\hline$\langle 28,28,37\rangle$ & 3360 & 840 & 2 & $\mathbb{Q}[-1,2,-3,5,-7]$ & $(2,2,2,2)$ & $\emptyset$ \\
$\langle 37,18,93\rangle$ & 13440 & 840 & 4 & & $(2,2,2,4)$ & $\emptyset$ \\
$\langle 37,24,72\rangle$ & 10080 & 280 & 6 & & $(2,2,2,4)$ & $\emptyset$ \\
\hline$\langle 8,8,167\rangle$ & 5280 & 1320 & 2 & $\mathbb{Q}[-1,2,-3,5,-11]$ & $(2,2,2,2)$ & $\emptyset$ \\
$\langle 32,16,167\rangle$ & 21120 & 1320 & 4 & & $(2,2,2,4)$ & $\emptyset$ \\
$\langle 32,24,87\rangle$ & 10560 & 660 & 4 & & $(2,2,2,4)$ & $\emptyset$ \\
\hline$\langle 41,38,41\rangle$ & 5280 & 1320 & 2 & $\mathbb{Q}[-1,2,-3,5,-11]$ & $(2,2,2,2)$ & $\emptyset$ \\
$\langle 41,6,129\rangle$ & 21120 & 1320 & 4 & & $(2,2,2,4)$ & $\emptyset$ \\
$\langle 41,10,65\rangle$ & 10560 & 660 & 4 & & $(2,2,2,4)$ & $\emptyset$
\end{tabular}

TABLE 3. Equivalence classes $C$ of forms $(\# \delta(C)=2, \# C=4)$

\begin{tabular}{cccc|cc|c}
$Q$ & $|D|$ & $|d|$ & $f$ & $P$ & $\mathrm{Cl}_{f}(d)$ & $E$ \\
\hline \hline$\langle 4,4,7\rangle$ & 96 & 24 & 2 & $\mathbb{Q}[-1,2,-3]$ & $(2,2)$ & $\emptyset$ \\
$\langle 7,6,15\rangle$ & 384 & 24 & 4 & & $(2,4)$ & $\emptyset$ \\
$\langle 7,2,7\rangle$ & 192 & 3 & 8 & & $(2,2)$ & $\emptyset$ \\
$\langle 7,4,28\rangle$ & 768 & 3 & 16 & & $(2,4)$ & $\emptyset$ \\
\hline$\langle 8,8,17\rangle$ & 480 & 120 & 2 & $\mathbb{Q}[-1,2,-3,5]$ & $(2,2,2)$ & $\emptyset$ \\
$\langle 17,16,32\rangle$ & 1920 & 120 & 4 & & $(2,2,4)$ & $\emptyset$ \\
$\langle 17,14,17\rangle$ & 960 & 15 & 8 & & $(2,2,2)$ & $\emptyset$ \\
$\langle 17,6,57\rangle$ & 3840 & 15 & 16 & & $(2,2,4)$ & $\emptyset$
\end{tabular}

TABLE 4. Equivalence classes $C$ of quadratic forms $(\# \delta(C)=3$, $\# C=3)$

\begin{tabular}{cccc|cc|c}
$Q$ & $|D|$ & $|d|$ & $f$ & $P$ & $\mathrm{Cl}_{f}(d)$ & $E$ \\
\hline \hline$\langle 1,0,24\rangle$ & 96 & 24 & 2 & $\mathbb{Q}[-1,2,-3]$ & $(2,2)$ & $\emptyset$ \\
$\langle 1,0,48\rangle$ & 192 & 3 & 8 & & $(2,2)$ & $\emptyset$ \\
$\langle 1,0,72\rangle$ & 288 & 8 & 6 & & $(2,2)$ & $\emptyset$ \\
\hline$\langle 7,4,52\rangle$ & 1440 & 40 & 6 & $\mathbb{Q}[-1,2,-3,5]$ & $(2,2,4)$ & $\emptyset$ \\
$\langle 7,6,87\rangle$ & 2400 & 24 & 10 & & $(2,2,4)$ & $\emptyset$ \\
$\langle 7,2,103\rangle$ & 2880 & 20 & 12 & & $(2,2,4)$ & $\emptyset$ \\
\hline$\langle 15,0,56\rangle$ & 3360 & 840 & 2 & $\mathbb{Q}[-1,2,-3,5,-7]$ & $(2,2,2,2)$ & $\emptyset$ \\
$\langle 39,12,44\rangle$ & 6720 & 420 & 4 & & $(2,2,2,4)$ & $\emptyset$ \\
$\langle 36,12,71\rangle$ & 10080 & 280 & 6 & & $(2,2,2,4)$ & $\emptyset$
\end{tabular}


TABle 5. Equivalence classes $C$ of quadratic forms $(\# \delta(C)=3$, $\# C=4)$

\begin{tabular}{cccc|cc|c}
$Q$ & $|D|$ & $|d|$ & $f$ & $P$ & $\mathrm{Cl}_{f}(d)$ & $E$ \\
\hline \hline$\langle 8,0,15\rangle$ & 480 & 120 & 2 & $\mathbb{Q}[-1,2,-3,5]$ & $(2,2,2)$ & $\emptyset$ \\
$\langle 12,12,23\rangle$ & 960 & 15 & 8 & & $(2,2,2)$ & $\emptyset$ \\
$\langle 23,22,47\rangle$ & 3840 & 15 & 16 & & $(2,2,4)$ & $\emptyset$ \\
$\langle 23,8,32\rangle$ & 2880 & 20 & 12 & & $(2,2,4)$ & $\emptyset$ \\
\hline$\langle 11,2,11\rangle$ & 480 & 120 & 2 & $\mathbb{Q}[-1,2,-3,5]$ & $(2,2,2)$ & $\emptyset$ \\
$\langle 11,4,44\rangle$ & 1920 & 120 & 4 & & $(2,2,4)$ & $\emptyset$ \\
$\langle 11,10,35\rangle$ & 1440 & 40 & 6 & & $(2,2,4)$ & $\emptyset$ \\
$\langle 11,8,56\rangle$ & 2400 & 24 & 10 & & $(2,2,4)$ & $\emptyset$ \\
\hline$\langle 8,8,23\rangle$ & 672 & 168 & 2 & $\mathbb{Q}[-1,2,-3,-7]$ & $(2,2,2)$ & $\emptyset$ \\
$\langle 23,16,32\rangle$ & 2688 & 168 & 4 & & $(2,2,4)$ & $\emptyset$ \\
$\langle 15,6,23\rangle$ & 1344 & 84 & 4 & & $(2,2,4)$ & $\emptyset$ \\
$\langle 23,4,44\rangle$ & 4032 & 7 & 24 & & $(2,2,4)$ & $\emptyset$
\end{tabular}

TABLE 6. Equivalence classes $C$ of quadratic forms $(\# \delta(C)=1)$

\begin{tabular}{cccc|cc|c}
$Q$ & $|D|$ & $|d|$ & $f$ & $P$ & $\mathrm{Cl}_{f}(d)$ & $E$ \\
\hline \hline$\langle 1,1,1\rangle$ & 3 & 3 & 1 & $\mathbb{Q}[-3]$ & $(1)$ & $\{3\}$ \\
$\langle 1,0,3\rangle$ & 12 & 3 & 2 & & $(1)$ & $\{3\}$ \\
$\langle 1,1,7\rangle$ & 27 & 3 & 3 & & $(1)$ & $\emptyset$ \\
\hline$\langle 1,0,1\rangle$ & 4 & 4 & 1 & $\mathbb{Q}[-1]$ & $(1)$ & $\{2\}$ \\
$\langle 1,0,4\rangle$ & 16 & 4 & 2 & & $(1)$ & $\emptyset$
\end{tabular}

TABLE 7. Orders of quadratic fields with class groups of type (1)

\begin{tabular}{ccc|ccc}
$|d|$ & $f$ & $|D|$ & $|d|$ & $f$ & $|D|$ \\
\hline \hline 3 & 1 & 3 & 8 & 1 & 8 \\
3 & 2 & 12 & 11 & 1 & 11 \\
3 & 3 & 27 & 19 & 1 & 19 \\
4 & 1 & 4 & 43 & 1 & 43 \\
4 & 2 & 16 & 67 & 1 & 67 \\
7 & 1 & 7 & 163 & 1 & 163 \\
7 & 2 & 28 & & &
\end{tabular}


TABLE 8. Orders of quadratic fields with class groups of type (2)

\begin{tabular}{ccc|ccc|ccc}
$|d|$ & $f$ & $|D|$ & $|d|$ & $f$ & $|D|$ & $|d|$ & $f$ & $|D|$ \\
\hline \hline 3 & 4 & 48 & 15 & 1 & 15 & 115 & 1 & 115 \\
3 & 5 & 75 & 15 & 2 & 60 & 123 & 1 & 123 \\
3 & 7 & 147 & 20 & 1 & 20 & 148 & 1 & 148 \\
4 & 3 & 36 & 24 & 1 & 24 & 187 & 1 & 187 \\
4 & 4 & 64 & 35 & 1 & 35 & 232 & 1 & 232 \\
4 & 5 & 100 & 40 & 1 & 40 & 235 & 1 & 235 \\
7 & 4 & 112 & 51 & 1 & 51 & 267 & 1 & 267 \\
8 & 2 & 32 & 52 & 1 & 52 & 403 & 1 & 403 \\
8 & 3 & 72 & 88 & 1 & 88 & 427 & 1 & 427 \\
11 & 3 & 99 & 91 & 1 & 91 & & &
\end{tabular}

TABLE 9. Orders of quadratic fields with class groups of type (4)

\begin{tabular}{ccc|ccc|ccc|ccc}
$|d|$ & $f$ & $|D|$ & $|d|$ & $f$ & $|D|$ & $|d|$ & $f$ & $|D|$ & $|d|$ & $f$ & $|D|$ \\
\hline \hline 3 & 11 & 363 & 39 & 1 & 39 & 184 & 1 & 184 & 723 & 1 & 723 \\
3 & 13 & 507 & 39 & 2 & 156 & 203 & 1 & 203 & 763 & 1 & 763 \\
4 & 6 & 144 & 43 & 3 & 387 & 219 & 1 & 219 & 772 & 1 & 772 \\
4 & 7 & 196 & 52 & 2 & 208 & 259 & 1 & 259 & 955 & 1 & 955 \\
4 & 8 & 256 & 55 & 1 & 55 & 291 & 1 & 291 & 1003 & 1 & 1003 \\
4 & 10 & 400 & 55 & 2 & 220 & 292 & 1 & 292 & 1027 & 1 & 1027 \\
7 & 3 & 63 & 56 & 1 & 56 & 323 & 1 & 323 & 1227 & 1 & 1227 \\
7 & 6 & 252 & 67 & 3 & 603 & 328 & 1 & 328 & 1243 & 1 & 1243 \\
8 & 4 & 128 & 68 & 1 & 68 & 355 & 1 & 355 & 1387 & 1 & 1387 \\
11 & 5 & 275 & 136 & 1 & 136 & 388 & 1 & 388 & 1411 & 1 & 1411 \\
19 & 3 & 171 & 148 & 2 & 592 & 568 & 1 & 568 & 1507 & 1 & 1507 \\
19 & 5 & 475 & 155 & 1 & 155 & 667 & 1 & 667 & 1555 & 1 & 1555 \\
20 & 2 & 80 & 163 & 3 & 1467 & & & & & &
\end{tabular}

TABLE 10. Orders of quadratic fields with class groups of type $(2,2)$

\begin{tabular}{ccc|ccc|ccc}
$|d|$ & $f$ & $|D|$ & $|d|$ & $f$ & $|D|$ & $|d|$ & $f$ & $|D|$ \\
\hline \hline 3 & 8 & 192 & 168 & 1 & 168 & 520 & 1 & 520 \\
7 & 8 & 448 & 195 & 1 & 195 & 532 & 1 & 532 \\
8 & 6 & 288 & 228 & 1 & 228 & 555 & 1 & 555 \\
15 & 4 & 240 & 232 & 2 & 928 & 595 & 1 & 595 \\
20 & 3 & 180 & 280 & 1 & 280 & 627 & 1 & 627 \\
24 & 2 & 96 & 312 & 1 & 312 & 708 & 1 & 708 \\
35 & 3 & 315 & 340 & 1 & 340 & 715 & 1 & 715 \\
40 & 2 & 160 & 372 & 1 & 372 & 760 & 1 & 760 \\
84 & 1 & 84 & 408 & 1 & 408 & 795 & 1 & 795 \\
88 & 2 & 352 & 435 & 1 & 435 & 1012 & 1 & 1012 \\
120 & 1 & 120 & 483 & 1 & 483 & 1435 & 1 & 1435 \\
132 & 1 & 132 & & & & & &
\end{tabular}


TABLE 11. Orders of quadratic fields with class groups of type $(2,4)$

\begin{tabular}{ccc|ccc|ccc|ccc|ccc}
$|d|$ & $f$ & $|D|$ & $|d|$ & $f$ & $|D|$ & $|d|$ & $f$ & $|D|$ & $|d|$ & $f$ & $|D|$ & $|d|$ & $f$ & $|D|$ \\
\hline \hline 3 & 16 & 768 & 84 & 2 & 336 & 308 & 1 & 308 & 987 & 1 & 987 & 2067 & 1 & 2067 \\
4 & 12 & 576 & 88 & 3 & 792 & 323 & 3 & 2907 & 1012 & 2 & 4048 & 2139 & 1 & 2139 \\
4 & 15 & 900 & 88 & 4 & 1408 & 328 & 2 & 1312 & 1032 & 1 & 1032 & 2163 & 1 & 2163 \\
4 & 20 & 1600 & 91 & 3 & 819 & 340 & 2 & 1360 & 1060 & 1 & 1060 & 2212 & 1 & 2212 \\
7 & 12 & 1008 & 91 & 5 & 2275 & 372 & 2 & 1488 & 1128 & 1 & 1128 & 2392 & 1 & 2392 \\
7 & 16 & 1792 & 115 & 3 & 1035 & 403 & 3 & 3627 & 1131 & 1 & 1131 & 2451 & 1 & 2451 \\
8 & 12 & 1152 & 132 & 2 & 528 & 427 & 3 & 3843 & 1204 & 1 & 1204 & 2632 & 1 & 2632 \\
11 & 15 & 2475 & 136 & 2 & 544 & 456 & 1 & 456 & 1240 & 1 & 1240 & 2667 & 1 & 2667 \\
20 & 4 & 320 & 148 & 3 & 1332 & 532 & 2 & 2128 & 1288 & 1 & 1288 & 2715 & 1 & 2715 \\
20 & 6 & 720 & 148 & 4 & 2368 & 552 & 1 & 552 & 1443 & 1 & 1443 & 2755 & 1 & 2755 \\
24 & 4 & 384 & 155 & 3 & 1395 & 564 & 1 & 564 & 1635 & 1 & 1635 & 2788 & 1 & 2788 \\
24 & 5 & 600 & 184 & 2 & 736 & 568 & 2 & 2272 & 1659 & 1 & 1659 & 2968 & 1 & 2968 \\
39 & 4 & 624 & 187 & 3 & 1683 & 580 & 1 & 580 & 1672 & 1 & 1672 & 3172 & 1 & 3172 \\
40 & 3 & 360 & 203 & 3 & 1827 & 616 & 1 & 616 & 1752 & 1 & 1752 & 3243 & 1 & 3243 \\
40 & 4 & 640 & 228 & 2 & 912 & 651 & 1 & 651 & 1768 & 1 & 1768 & 3355 & 1 & 3355 \\
51 & 5 & 1275 & 232 & 3 & 2088 & 708 & 2 & 2832 & 1771 & 1 & 1771 & 3507 & 1 & 3507 \\
52 & 3 & 468 & 232 & 4 & 3712 & 820 & 1 & 820 & 1780 & 1 & 1780 & 4123 & 1 & 4123 \\
52 & 4 & 832 & 235 & 3 & 2115 & 852 & 1 & 852 & 1947 & 1 & 1947 & 4323 & 1 & 4323 \\
55 & 4 & 880 & 260 & 1 & 260 & 868 & 1 & 868 & 1992 & 1 & 1992 & 5083 & 1 & 5083 \\
56 & 2 & 224 & 264 & 1 & 264 & 915 & 1 & 915 & 2020 & 1 & 2020 & 5467 & 1 & 5467 \\
56 & 3 & 504 & 276 & 1 & 276 & 952 & 1 & 952 & 2035 & 1 & 2035 & 6307 & 1 & 6307 \\
68 & 3 & 612 & & & & & & & & & & &
\end{tabular}

TABLE 12. Orders of quadratic fields with class groups of type $(2,2,2)$

\begin{tabular}{ccc|ccc}
$|d|$ & $f$ & $|D|$ & $|d|$ & $f$ & $|D|$ \\
\hline \hline 15 & 8 & 960 & 1092 & 1 & 1092 \\
120 & 2 & 480 & 1155 & 1 & 1155 \\
168 & 2 & 672 & 1320 & 1 & 1320 \\
280 & 2 & 1120 & 1380 & 1 & 1380 \\
312 & 2 & 1248 & 1428 & 1 & 1428 \\
408 & 2 & 1632 & 1540 & 1 & 1540 \\
420 & 1 & 420 & 1848 & 1 & 1848 \\
520 & 2 & 2080 & 1995 & 1 & 1995 \\
660 & 1 & 660 & 3003 & 1 & 3003 \\
760 & 2 & 3040 & 3315 & 1 & 3315 \\
840 & 1 & 840 & & &
\end{tabular}


TABLE 13. Orders of quadratic fields with class groups of type $(2,2,4)$

\begin{tabular}{ccc|ccc|ccc|ccc|ccc}
$|d|$ & $f$ & $|D|$ & $|d|$ & $f$ & $|D|$ & $|d|$ & $f$ & $|D|$ & $|d|$ & $f$ & $|D|$ & $|d|$ & $f$ & $|D|$ \\
\hline \hline 7 & 24 & 4032 & 372 & 4 & 5952 & 1288 & 2 & 5152 & 3432 & 1 & 3432 & 6708 & 1 & 6708 \\
15 & 16 & 3840 & 408 & 4 & 6528 & 1380 & 2 & 5520 & 3480 & 1 & 3480 & 6820 & 1 & 6820 \\
20 & 12 & 2880 & 420 & 2 & 1680 & 1428 & 2 & 5712 & 3588 & 1 & 3588 & 6820 & 1 & 6820 \\
24 & 10 & 2400 & 456 & 2 & 1824 & 1435 & 3 & 12915 & 3640 & 1 & 3640 & 7315 & 1 & 7315 \\
39 & 8 & 2496 & 520 & 3 & 4680 & 1540 & 2 & 6160 & 3795 & 1 & 3795 & 7395 & 1 & 7395 \\
40 & 6 & 1440 & 520 & 4 & 8320 & 1560 & 1 & 1560 & 3828 & 1 & 3828 & 7480 & 1 & 7480 \\
55 & 8 & 3520 & 532 & 3 & 4788 & 1672 & 2 & 6688 & 4020 & 1 & 4020 & 7540 & 1 & 7540 \\
56 & 6 & 2016 & 532 & 4 & 8512 & 1716 & 1 & 1716 & 4180 & 1 & 4180 & 7755 & 1 & 7755 \\
84 & 4 & 1344 & 552 & 2 & 2208 & 1752 & 2 & 7008 & 4260 & 1 & 4260 & 7995 & 1 & 7995 \\
84 & 5 & 2100 & 595 & 3 & 5355 & 1768 & 2 & 7072 & 4420 & 1 & 4420 & 8008 & 1 & 8008 \\
88 & 6 & 3168 & 616 & 2 & 2464 & 1860 & 1 & 1860 & 4440 & 1 & 4440 & 8052 & 1 & 8052 \\
120 & 4 & 1920 & 660 & 2 & 2640 & 1992 & 2 & 7968 & 4452 & 1 & 4452 & 8547 & 1 & 8547 \\
132 & 4 & 2112 & 708 & 4 & 11328 & 2040 & 1 & 2040 & 4488 & 1 & 4488 & 8680 & 1 & 8680 \\
168 & 4 & 2688 & 715 & 3 & 6435 & 2244 & 1 & 2244 & 4515 & 1 & 4515 & 8715 & 1 & 8715 \\
228 & 4 & 3648 & 760 & 3 & 6840 & 2280 & 1 & 2280 & 4740 & 1 & 4740 & 8835 & 1 & 8835 \\
232 & 6 & 8352 & 760 & 4 & 12160 & 2392 & 2 & 9568 & 5115 & 1 & 5115 & 8932 & 1 & 8932 \\
260 & 3 & 2340 & 952 & 2 & 3808 & 2436 & 1 & 2436 & 5160 & 1 & 5160 & 9867 & 1 & 9867 \\
264 & 2 & 1056 & 1012 & 3 & 9108 & 2580 & 1 & 2580 & 5187 & 1 & 5187 & 10948 & 1 & 10948 \\
280 & 3 & 2520 & 1012 & 4 & 16192 & 2632 & 2 & 10528 & 5208 & 1 & 5208 & 11067 & 1 & 11067 \\
280 & 4 & 4480 & 1032 & 2 & 4128 & 2760 & 1 & 2760 & 5412 & 1 & 5412 & 11715 & 1 & 11715 \\
308 & 3 & 2772 & 1092 & 2 & 4368 & 2968 & 2 & 11872 & 6195 & 1 & 6195 & 13195 & 1 & 13195 \\
312 & 4 & 4992 & 1128 & 2 & 4512 & 3108 & 1 & 3108 & 6420 & 1 & 6420 & 14763 & 1 & 14763 \\
340 & 3 & 3060 & 1140 & 1 & 1140 & 3192 & 1 & 3192 & 6580 & 1 & 6580 & 16555 & 1 & 16555 \\
340 & 4 & 5440 & 1240 & 2 & 4960 & 3220 & 1 & 3220 & 6612 & 1 & 6612 & &
\end{tabular}

TABLE 14. Orders of quadratic fields with class groups of type $(2,2,2,2)$

\begin{tabular}{ccc}
$|d|$ & $f$ & $|D|$ \\
\hline \hline 840 & 2 & 3360 \\
1320 & 2 & 5280 \\
1848 & 2 & 7392 \\
5460 & 1 & 5460
\end{tabular}

TABLE 15. Orders of quadratic fields with class groups of type $(2,2,2,4)$

\begin{tabular}{ccc|ccc|ccc}
$|d|$ & $f$ & $|D|$ & $|d|$ & $f$ & $|D|$ & $|d|$ & $f$ & $|D|$ \\
\hline \hline 280 & 6 & 10080 & 2280 & 2 & 9120 & 8680 & 2 & 34720 \\
420 & 4 & 6720 & 2760 & 2 & 11040 & 9240 & 1 & 9240 \\
520 & 6 & 18720 & 3192 & 2 & 12768 & 10920 & 1 & 10920 \\
660 & 4 & 10560 & 3432 & 2 & 13728 & 12180 & 1 & 12180 \\
760 & 6 & 27360 & 3480 & 2 & 13920 & 14280 & 1 & 14280 \\
840 & 4 & 13440 & 3640 & 2 & 14560 & 14820 & 1 & 14820 \\
1092 & 4 & 17472 & 4440 & 2 & 17760 & 17220 & 1 & 17220 \\
1320 & 4 & 21120 & 4488 & 2 & 17952 & 19320 & 1 & 19320 \\
1380 & 4 & 22080 & 5160 & 2 & 20640 & 19380 & 1 & 19380 \\
1428 & 4 & 22848 & 5208 & 2 & 20832 & 19635 & 1 & 19635 \\
1540 & 3 & 13860 & 5460 & 2 & 21840 & 20020 & 1 & 20020 \\
1540 & 4 & 24640 & 7140 & 1 & 7140 & 31395 & 1 & 31395 \\
1560 & 2 & 6240 & 7480 & 2 & 29920 & 33915 & 1 & 33915 \\
1848 & 4 & 29568 & 8008 & 2 & 32032 & 40755 & 1 & 40755 \\
2040 & 2 & 8160 & 8580 & 1 & 8580 & & &
\end{tabular}


TABLE 16. Orders of quadratic fields with class groups of type $(2,2,2,2,4)$

\begin{tabular}{ccc}
$|d|$ & $f$ & $|D|$ \\
\hline \hline 5460 & 4 & 87360 \\
9240 & 2 & 36960 \\
10920 & 2 & 43680 \\
14280 & 2 & 57120 \\
19320 & 2 & 77280
\end{tabular}

\section{REFERENCES}

[BS] Z.I. Borevich and I.R. Shafarevich, Number theory, Academic Press, New York, 1966. MR0195803 (33:4001)

[Ch] S. Chowla, An extension of Heilbronn's class number theorem, Quart. J. Math. Oxford Ser. 5 (1934), 304-307.

[Cox] David A. Cox, Primes of the form $x^{2}+n y^{2}$ : Fermat, class field theory, and complex multiplication, John Wiley \& Sons, Inc., New York, 1989. MR.1028322 (90m:11016)

[D] Harold Davenport, Multiplicative number theory, third ed., Graduate texts in mathematics, vol. 74, Springer-Verlag, New York, 2000. MR.1790423 (2001f:11001)

[J] Gerald Janusz, Algebraic number fields, second ed., Graduate studies in mathematics, vol. 7, American Mathematical Society, Providence, RI, 1996. MR.1362545 (96j:11137)

[JK] William C. Jagy and Irving Kaplansky, Positive definite binary quadratic forms that represent the same primes, preprint.

[La] Serge Lang, Algebraic number theory, 2nd ed., Graduate studies in mathematics, vol. 110, Berlin: Springer-Verlag, 1994. MR 1282723 (95f:11085)

[Lo] Stéphane Louboutin, Minorations (sous l'hypothèse de Riemann généralisée) des nombres de classes de corps quadratiques imaginaires. Application, C. R. Acad. Sci. Paris Sér. I Math. 310 (1990), 795-800. MR1058499 (91e:11126)

[N] Jürgen Neukirch, Algebraic number theory, Grundlehren der mathematischen Wissenschaften, vol. 322, Springer-Verlag, Berlin, 1999. MR.1697859 (2000m:11104)

[S] C.L. Siegel, Über die Classenzahl quadratischer Zahlkörper, Acta Arith. 1 (1935), 83-86.

[T] Tikao Tatuzawa, On a theorem of Siegel, Japan. J. Math. 21 (1951), 163-178. MR0051262 $(14: 452 \mathrm{c})$

[Wa] Lawrence C. Washington, Introduction to cyclotomic fields, second ed., Graduate texts in Mathematics, vol. 83, Springer-Verlag, New York, 1997. MR.1421575 (97h:11130)

[We] P.J. Weinberger, Exponents of the class groups of complex quadratic fields, Acta Arith. 22 (1973), 117-124. MR0313221 (47:1776)

Department of Mathematics, University of California, Berkeley, Berkeley, CaliFORNIA 94720

Current address: Institute for Mathematics and its Applications, 400 Lind Hall, 237 Church Street, University of Minnesota, Minneapolis, Minnesota 55455

E-mail address: jvoight@gmail.com 